\title{
LA INFLUENGIA DEL PAISAJE SONORO EN LA GALIDAD DEL ENTORNO URBANO; UN ENFOQUE HACIA LA EXPERIENCIA SONORA DE LA CIUDAD LATINOAMERICANA
}

\section{THE INFLUENGE OF THE SOUNDSCAPE ON THE QUALTYY OF THE URBAN ENVIRONMENT; AN APPROACH TO THE SOUND EXPERIENCE OF THE LATIN AMERICAN GITY}

\author{
JULIÁN GRIJALBA \\ Arquitecto Investigador \\ Facultad de Arte \& Diseño \\ Centro de Estudios Urbanos - Institución Universitaria Colegio \\ Mayor Del Cauca \\ Carrera 5 \# 5 - 40 Claustro de la Encarnación (Colombia) \\ Teléfono: 5728240562 ext. 121 \\ Email: directorceu@unimayor.edu.co
}

\section{PALABRAS CLAVES}

Paisaje Sonoro, Ruido Urbano, Cartografias Sonoras, Entorno Acústico Urbano,

Planificación Urbana Sostenible, Sistemas de Información Geográfica.

\section{KEY WORDS}

Soundscape, Urban Noise, Sound Cartographies, Urban Acoustic

Environment, Sustainable Urban Planning, Geographic Information Systems. 


\section{Resumen}

El entorno acústico urbano está sujeto a características físicas, culturales y perceptuales del lugar, en ese sentido la exploración del paisaje sonoro es de importancia para comprender el universo sonoro de la ciudad desde un enfoque amplio e integral. Esta investigación examinó la influencia de los aspectos físicos y perceptuales del paisaje sonoro en la calidad de los entornos acústicos de una pieza urbana del sector histórico de la ciudad de Popayán -Colombia.

Se realizaron 121 mediciones de ruido y 50 grabaciones estereofónicas con el fin de obtener mapas de ruido y del paisaje sonoro urbano que permitieron evaluar el fenómeno sonoro de la ciudad desde diferentes puntos de vista. Los resultados mostraron la relación entre los patrones de comportamiento del paisaje sonoro y los niveles de presión sonora producidos por la actividad urbana, así como también se encontraron entornos acústicos cambiantes a nivel espaciotemporal. Los datos sugieren que las variaciones en la composición del paisaje sonoro y los indicadores acústicos del ruido (dBLeq, dBMin, dBMax) podrían influir significativamente en la experiencia sonora de la ciudad y por tanto en la calidad del entorno urbano.

Finalmente, se destaca la importancia del paisaje sonoro como instrumento de planificación urbana sostenible, ya a que permite que los tomadores de decisiones puedan aunar esfuerzos en el mejoramiento de la calidad del entorno acústico urbano mediante la inclusión de áreas urbanas que propicien paisajes sonoros equilibrados y modelos de ocupación territorial que contribuyan al bienestar social de la población. 


\section{Abstract}

The urban acoustic environment is subject to physical, cultural and perceptual factors of the place. Consequently, the exploration of soundscape is important to understand the sound universe of the city from a broad and integral approach. This research examined the influence of the physical and perceptual aspects of soundscape on the acoustic environments quality of an urban piece of Popayán historic sector (Colombia).

Thus, 121 noise measurements and 50 stereophonic recordings were made in order to obtain noise maps and urban soundscape maps that allowed evaluating the sound phenomenon of the city from different points of view. The results showed the relationship between soundscape behavior patterns and sound pressure levels by urban activity, as well as acoustic changes at a spatiotemporal level. The data that acoustic noise changes (dBLeq, $d B M i n, d B M a x)$ can influence the sound experience of the city and therefore the quality of the urban environment.

Finally, the importance of soundscape as an instrument of sustainable urban planning is highlighted, since it allows decision makers to improve the quality of the urban acoustic environment through the inclusion of urban areas that propitiate balanced soundscape and models of territorial occupation that contributes to the social welfare of the population. 


\section{Introducción}

La producción sonora de millones de asentamientos urbanos alrededor del mundo constituye el universo sonoro de la humanidad y a pesar de su notable afectación en las dinámicas urbanas, es solo en los años sesenta cuando las condiciones del entorno acústico se incorporan en el ejercicio de la planificación territorial (Raimbault y Dubois, 2005). Más adelante hacia la década de los noventa el carácter global de la problemática es reconocido, fijando así los límites de emisión de ruido indispensables para contrarrestar el deterioro en la salud pública y velar por el bienestar social de la población (Organización Mundial de la Salud, 1999; Stanners y Bourdeau, 1995).

En los últimos años se ha teorizado la ciudad a partir de la concepción y los modos de habitar el lugar, dejando de lado la complejidad del espacio sonoro como un importante componente sensorial que revela la relación de los habitantes urbanos con su mundo exterior (Agnew, 2011; Simmel, 1998). En ese sentido el tratamiento de lo sonoro a escala urbana ha sido enfocado principalmente en el mapeo del ruido, evaluando solo el aspecto físico del sonido (Can, Leclercqa y Lelongb, 2008; Geraghtya y O'Mahony, 2016; Hao y Kang, 2014; Stoter, Kluijver \& Kurakula, 2008; Tsai, Lin y Chen, 2009). Sin embargo replantearse una perspectiva más amplia respecto al sonido de la ciudad, radica justamente en el aporte que hace el fenómeno sonoro en la descripción de la vida urbana, esa interacción entre el medio sonoro y la ciudadanía que es descrita como paisaje sonoro (Jennings y Cain, 2013).

Así, el concepto multidisciplinar del paisaje sonoro es definido como todos los sonidos de un entorno en particular que son percibidos por el ser humano (Schafer, 1993). Aporte teórico de finales del siglo pasado que puso en relevancia los riesgos que podría representar la gran cloaca sonora del futuro, entendida como aquel entorno acústico configurado por objetos sonoros de escaso valor cultural para los oyentes (Schaeffer et al., 1967; Schafer, 1993; Truax, 2000). Actualmente el enfoque emergente del paisaje sonoro que prioriza aspectos del medio sonoro como la relación con el contexto, las emociones y la escucha humana, más allá de la ambigua noción que del ruido se tiene, ha sido tratado ampliamente en diversas investigaciones (Bruce y Davies, 2014; Davies et al. 2013; Hong y Jeon, 2017; Kang et al., 2016; Rehan, 2016).

El concepto del paisaje sonoro urbano comúnmente hace referencia a la percepción del conjunto de señales acústicas de un entorno urbano delimitado temporalmente (Liu y Kang, 2016). En ese sentido se puntualizó lo siguiente, en primer lugar el paisaje sonoro está directamente relacionado a la percepción humana y no a los parámetros acústicos del sonido, puesto que lógicamente es el ser humano quien moldea el sonido según sus atributos psicológicos (Jeon y Hong, 2015). De esta forma la gestión del paisaje sonoro en la ciudad integra la visión general del confort urbano, la calidad del entorno acústico en sí y la evaluación eficaz del entorno acústico percibido, tanto en función de una escala de determinación espacial como de un tiempo específico para su respectivo análisis semántico (Leus y Herssens, 2015).

En segundo lugar los parámetros físicos del sonido no son suficientes para comprender la forma en que los seres humanos perciben el paisaje sonoro de la ciudad (Watts y Pheasant, 2015). Más aun sabiendo que 
cuando los niveles LAeq son excesivamente altos, las intervenciones como barreras visuales o la reproducción de sonidos naturales mediante altavoces pierden total eficacia en el control del ruido urbano (Cerwen, 2016). Por tanto es premeditado afirmar que la reducción de los niveles de ruido está relacionada con la mejora de las condiciones ambientales de la ciudad (Yang y Kang, 2005).

En último lugar la experiencia sonora del ciudadano está determinada por la constante modificación del entorno urbano, así como también por el nivel de elocuencia del paisaje sonoro con su contexto urbano adyacente (Carles, Barrio y De Lucio, 1999; Mohammadi, 2009). De hecho, algunos estudios se han dado a la tarea de buscar relaciones entre el contexto urbano especifico y el paisaje sonoro resultante, perfilándose como un instrumento de recuperación de futuras configuraciones urbanas (Bahal y Tamer-Bayazit, 2017).

Acorde a la conceptualización expuesta hasta aquí, se definió que los parámetros acústicos del sonido son insuficientes en el tratamiento del ruido urbano en donde factores físicos, sociales y territoriales se encuentran involucrados (Liu, Kang, Luo, Behm y Coppack, 2013). Con lo anteriormente expuesto el presente estudio traslada la discusión teórica sobre el paisaje sonoro a la realidad latinoamericana en general y al contexto de la ciudad colombiana en particular, enmarcándose en la idea del paisaje sonoro como mecanismo de afectación del entorno urbano.

\section{Objetivo}

Examinar la influencia de la variabilidad espaciotemporal del paisaje sonoro en la calidad de los entornos acústicos de una pieza urbana de la ciudad de Popayán - Colombia, prestando particular atención al vínculo de la experiencia sonora del habitante de la ciudad con el ruido ambiental y el contexto urbano adyacente.

\section{Metodología}

El presente estudio de caso sobre el paisaje sonoro urbano empleó métodos cuantitativos y cualitativos en función de los factores más relevantes que conciernen al espacio sonoro de la ciudad. La pieza urbana delimitada en este trabajo permitió la elaboración de cartografías de ruido basadas tanto en indicadores acústicos como en puntuaciones de la sonoridad percibida del entorno acústico urbano. Toda la información obtenida fue contrastada mediante mapas sonoros a partir de lo cual se plateó la respectiva discusión teórica sobre el papel de la dimensión sonora en los procesos de planificación urbana.

\section{Recolección y análisis de datos}

\section{1 Área de estudio}

La unidad de gestión urbanística de Santo Domingo delimitada en el Plan Especial de Manejo y Protección (2010) del sector histórico del municipio de Popayán fue la pieza urbana seleccionada para el desarrollo de este trabajo. Principalmente se tuvieron en cuenta los contextos urbanos diferenciados del área de estudio como zonas comerciales, conjuntos residenciales, parques urbanos, oficinas y universidades, de importancia para examinar la dependencia espaciotemporal del paisaje sonoro urbano, ver figura 1. 


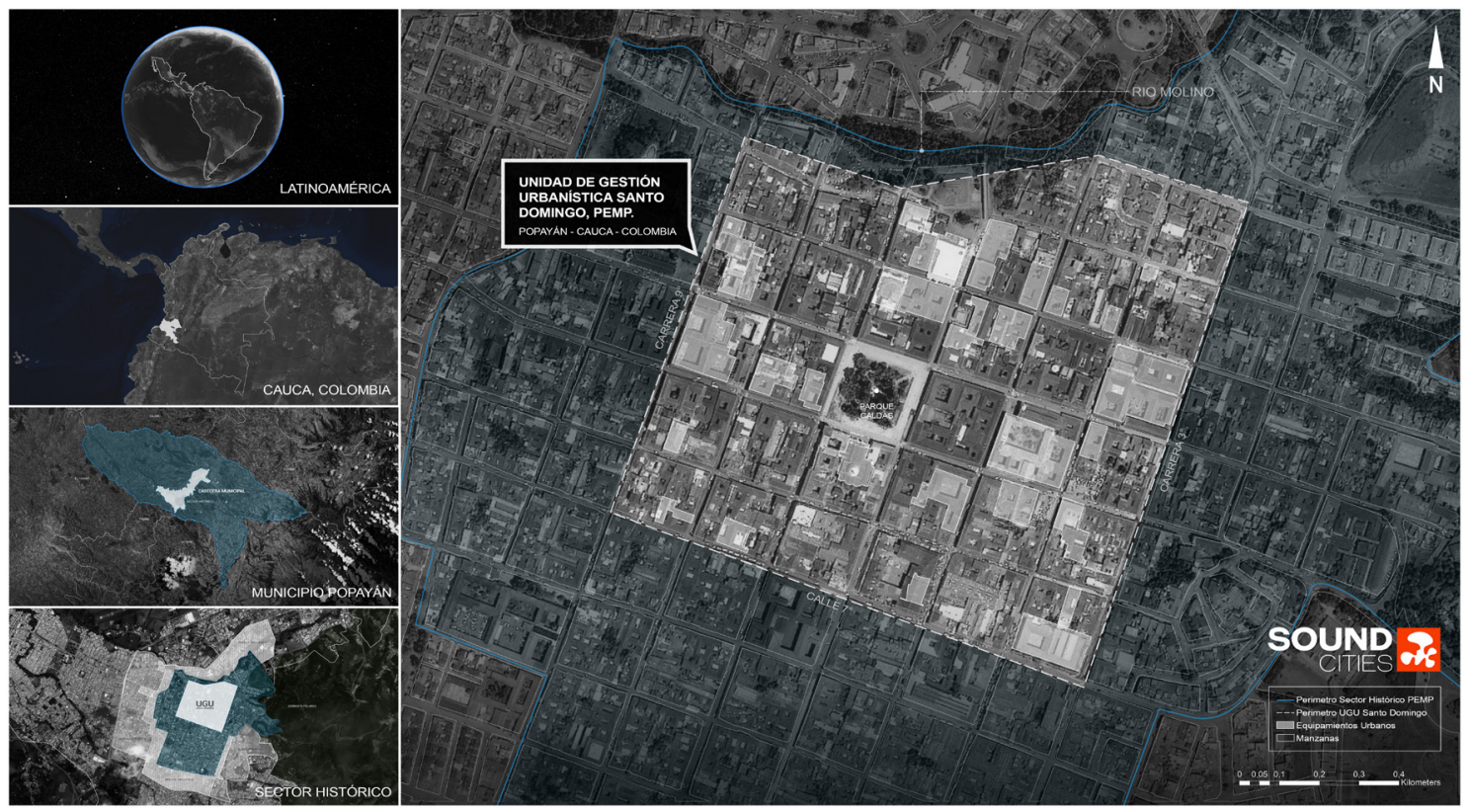

Figura 1: Unidad de gestión urbanística de Santo Domingo según Plan Especial de Manejo y Protección del sector histórico de Popayán - PEMP.

Elaboración: Centro de Estudios Urbanos, Colombia.

\subsection{Datos de ruido}

Una malla de muestreo de $50 \mathrm{~m} \times 50 \mathrm{~m}$ distribuida en ciento veintiún puntos fue utilizada para asegurar la medición del ruido en el área de estudio, ver figura 2. Los descriptores acústicos utilizados fueron el nivel equivalente, nivel mínimo y nivel máximo de presión sonora $\left(\mathrm{dB}_{\text {Leq, }} \mathrm{dB}_{\text {Min, }} \mathrm{dB}_{\text {Max). }}\right.$ Las mediciones fueron tomadas durante horarios diurnos en el mes de octubre del año 2016 entre las 8:00 a.m. y las 6:00 p.m. 


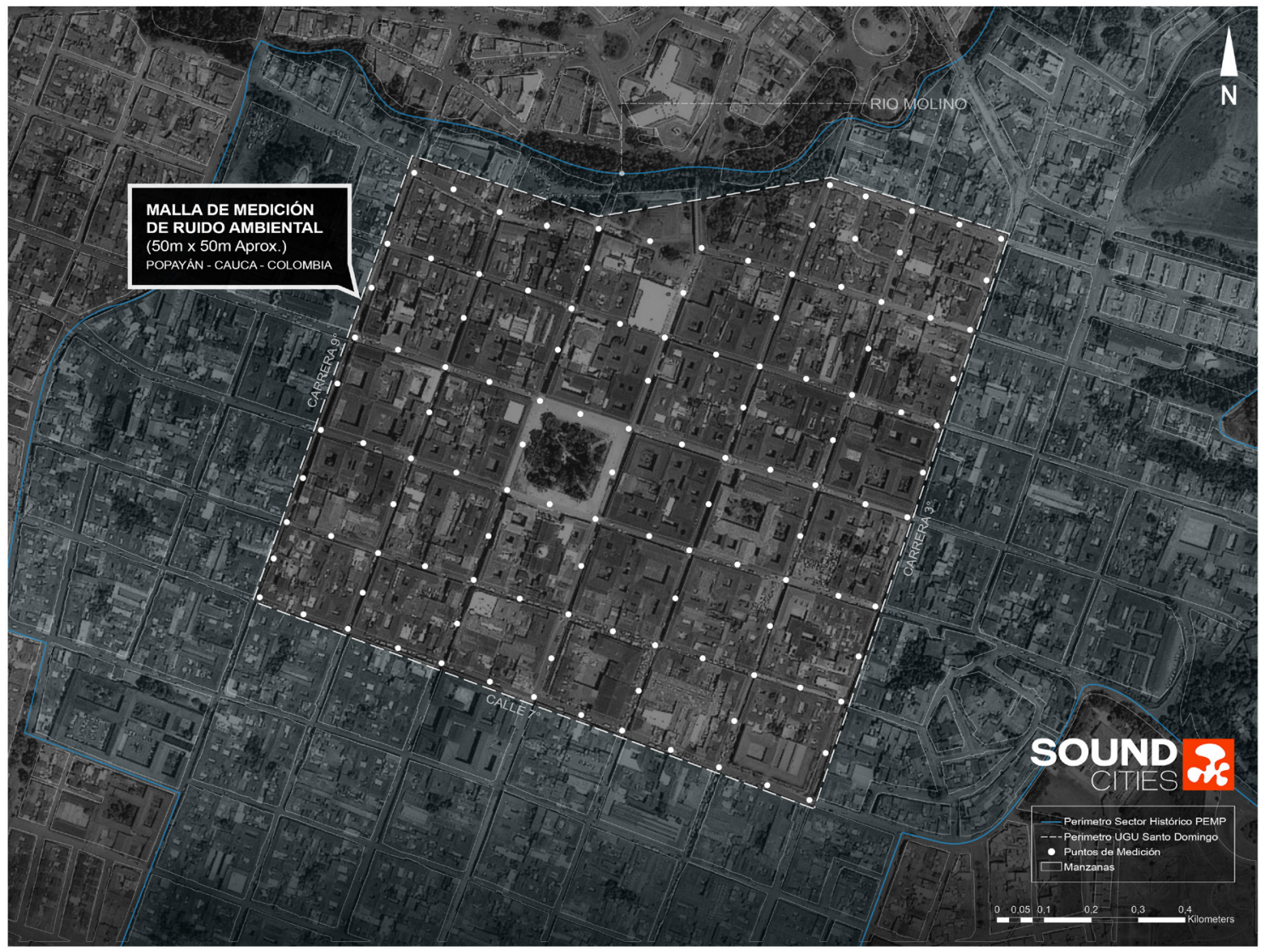

Figura 2: Distribución espacial de los puntos de medición de ruido. Elaboración: Centro de Estudios Urbanos, Colombia.

Respecto al tiempo de medición se fijó una duración de 15 minutos de registro por punto de muestreo, en donde se tuvieron en cuenta las técnicas de mapeo del ruido que utilizaron tiempos de registro similares (Geraghty y O'mahonyb, 2016; Zannin, Engel, Fiedler y Bunn, 2013), ver figura 3. Las mediciones fueron tomadas a una distancia de $1.5 \mathrm{~m}$ de suelo y se usó un sonómetro Sound Pro DL con filtros de 1/3 de octava.

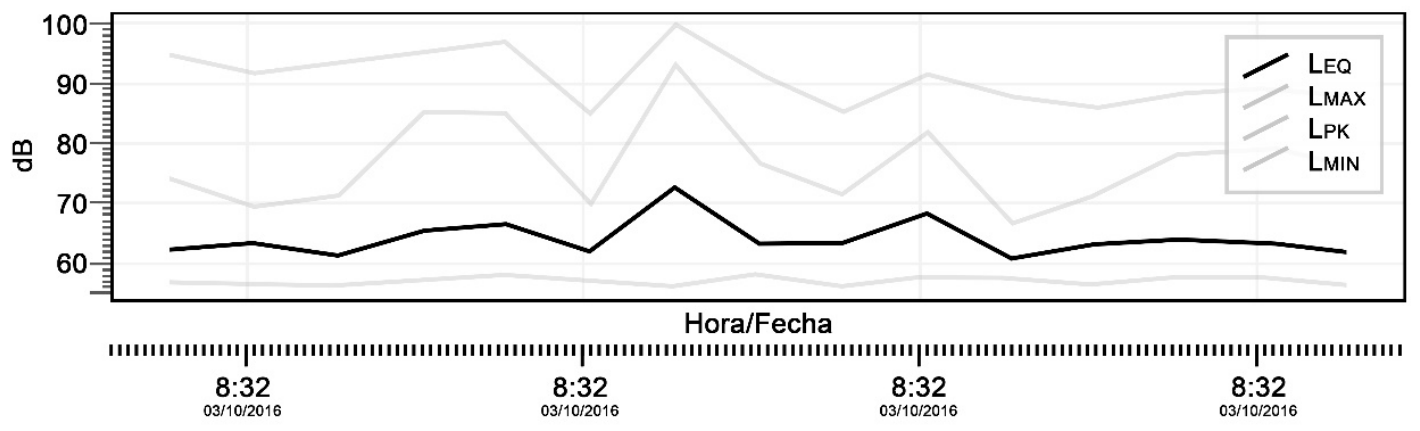

Figura 3: Datos de registro obtenidos durante 15 minutos de medición. Imagen obtenida de Detection Management Software DMS. 


\subsection{Datos del paisaje sonoro}

Un factor fundamental en la evaluación del paisaje sonoro es la captura de los sonidos producidos tanto por las actividades humanas como por el ambiente natural (Raimbault y Dubois, 2005). Los estudios que han intentado clasificar los eventos sonoros del entorno urbano acorde al significado de las fuentes que los produce, coinciden en su mayoría en que el paisaje sonoro puede agruparse por clases principales y estas a su vez desfragmentarse en subclases respectivamente (Pijanowski, Farina, Gage, Dumyahn y Krause, 2011).

La taxonomía del paisaje sonoro caracteriza las fuentes de sonido de un entorno urbano con base en dos criterios fundamentales; En primer lugar, que se pudiera aplicar en todo tipo de entornos acústicos, y en segundo lugar, la nomenclatura de fuentes sonoras debe ser seleccionada cuidadosamente para evitar juicios de valor con respecto a la percepción de las fuentes sonoras en el lugar (Brown, Kang y Gjestland, 2011). Conforme a la estandarización propuesta, la taxonomía del paisaje sonoro fue ajustada al contexto específico de la pieza urbana objeto de estudio de esta investigación, ver figura 4.

\section{ENTORNO ACÚSTICO \\ URBANO}

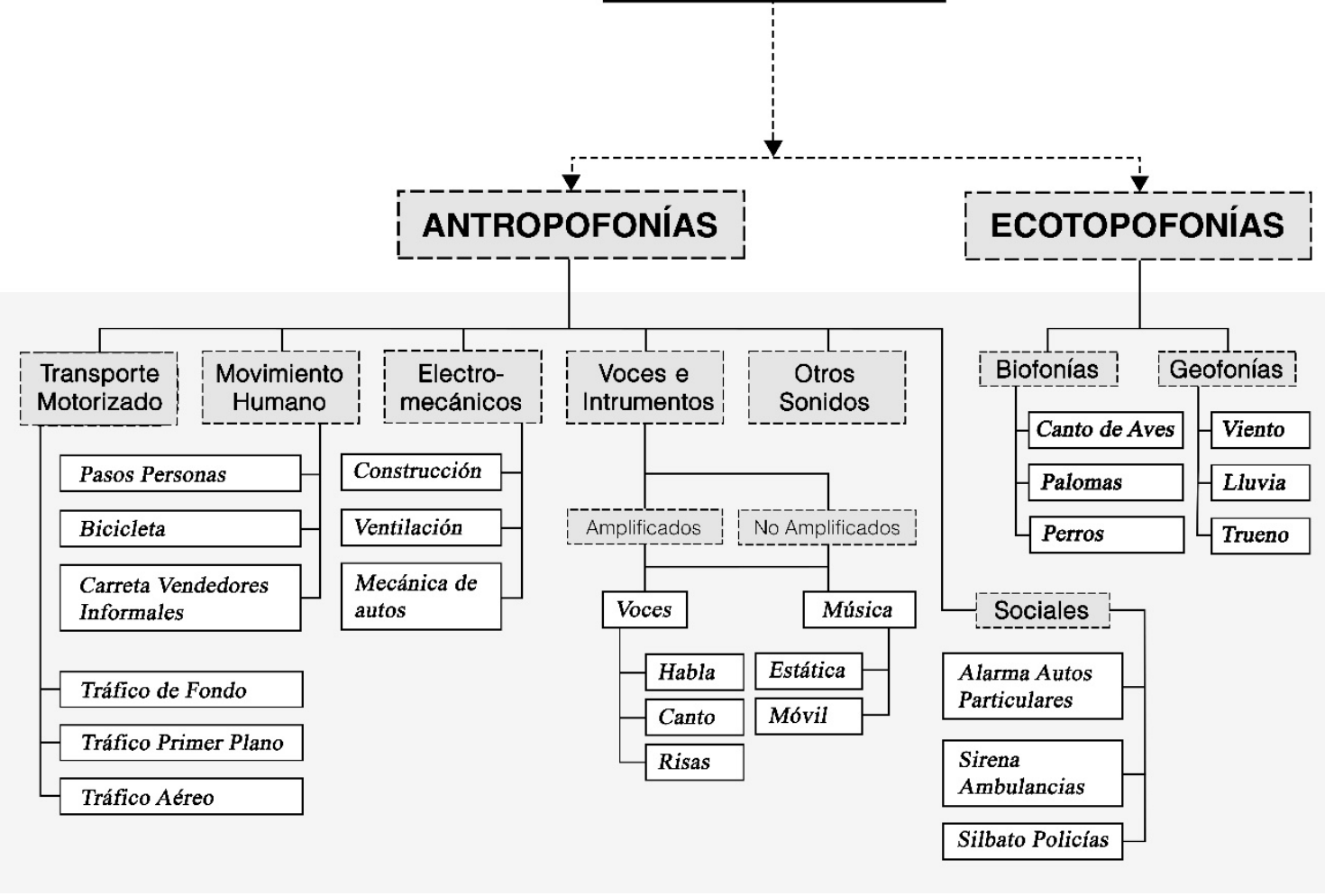

Figura 4: Taxonomía del paisaje sonoro ajustada al contexto del presente estudio. Elaboración propia.

Una vez ajustada la taxonomía del paisaje sonoro urbano, una malla de muestreo de $50 \mathrm{~m} \times 50 \mathrm{~m}$ se distribuyó en cincuenta puntos en la misma área en que se registraron las mediciones de ruido, ver figura 5. 
Asegurando así la grabación estereofónica de todos los puntos de muestreo con sus respectivos registros de sonoridad percibida en campo.

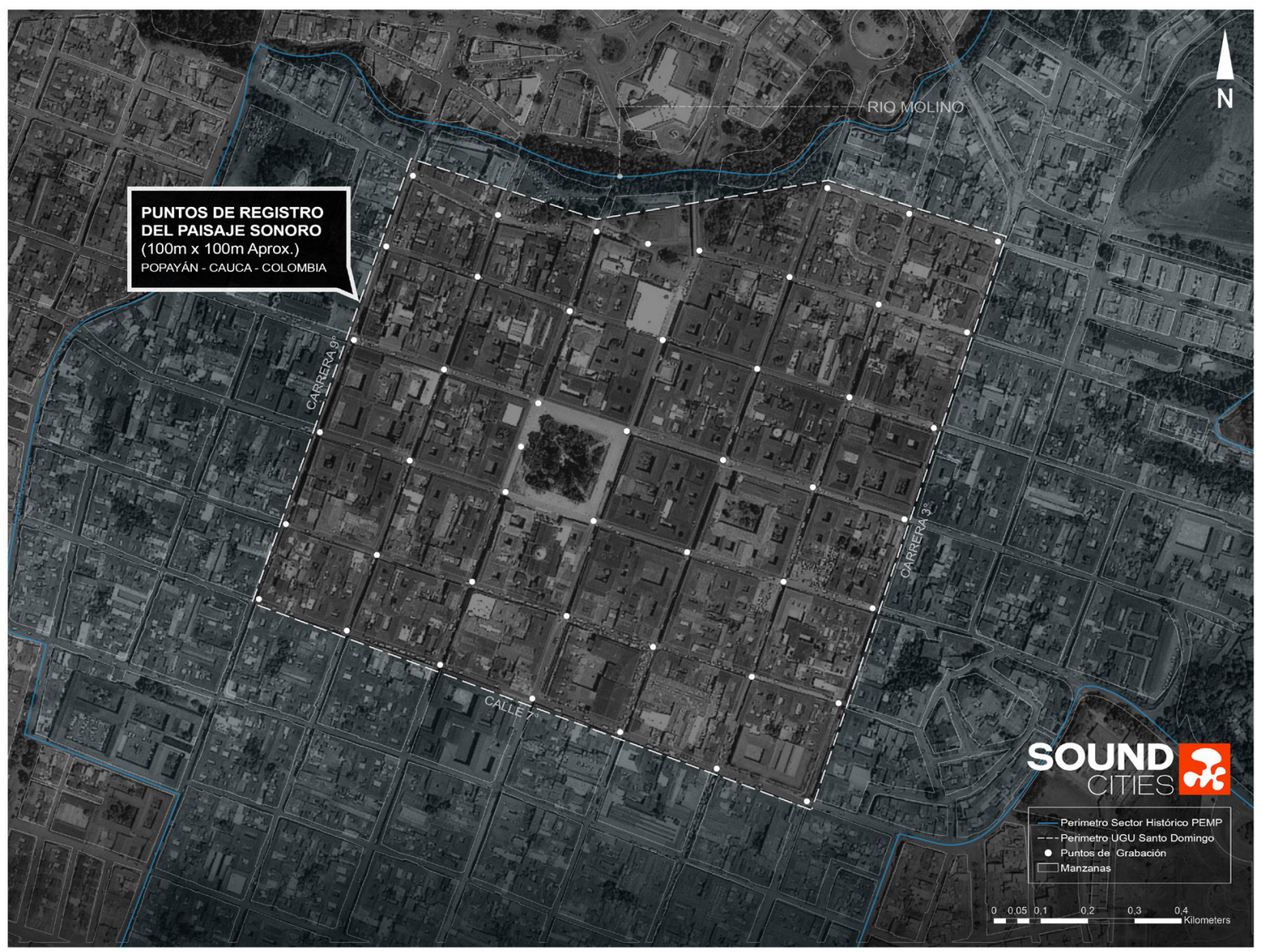

Figura 5: Distribución espacial de los puntos de grabación del paisaje sonoro. Elaboración: Centro de Estudios Urbanos, Colombia.

El trabajo se ejecutó durante el mes de febrero del año 2017, para cada punto de la malla de muestreo se registraron 10 minutos de paisaje sonoro durante cinco periodos sucesivos de dos horas de duración respectivamente (1er período: 08:00 - 10:00, $2^{\circ}$ período: 10:00 - 12: 00, $3^{\circ}$ período: 12:00 - 14:00, $4^{\circ}$ período: 14:00 - 16:00, $5^{\circ}$ período: 16: 00 - 18: 00). En paralelo grabaciones estereofónicas fueron realizadas mediante el sistema de suspensión de viento RODE-Blimp, un micrófono cardiode Sennheiser MKH-416 y una grabadora de alta calidad Tascam DR-40.

De forma similar al estudio sobre variabilidad del paisaje sonoro urbano (Liu et al., 2013), se segmentaron los 10 minutos de muestreo en veinte etapas de tiempo secuenciales con duración de 30 segundos cada una, de esta forma cada sonido de subclase propuesto en la taxonomía del paisaje sonoro fue calificado acorde a la sonoridad percibida en campo, para lo cual se usó una escala lineal de cinco puntos $(1=$ muy bajo, $2=$ bajo, $3=$ normal, $4=$ alto, $5=$ muy alto), ver figura 6 . 


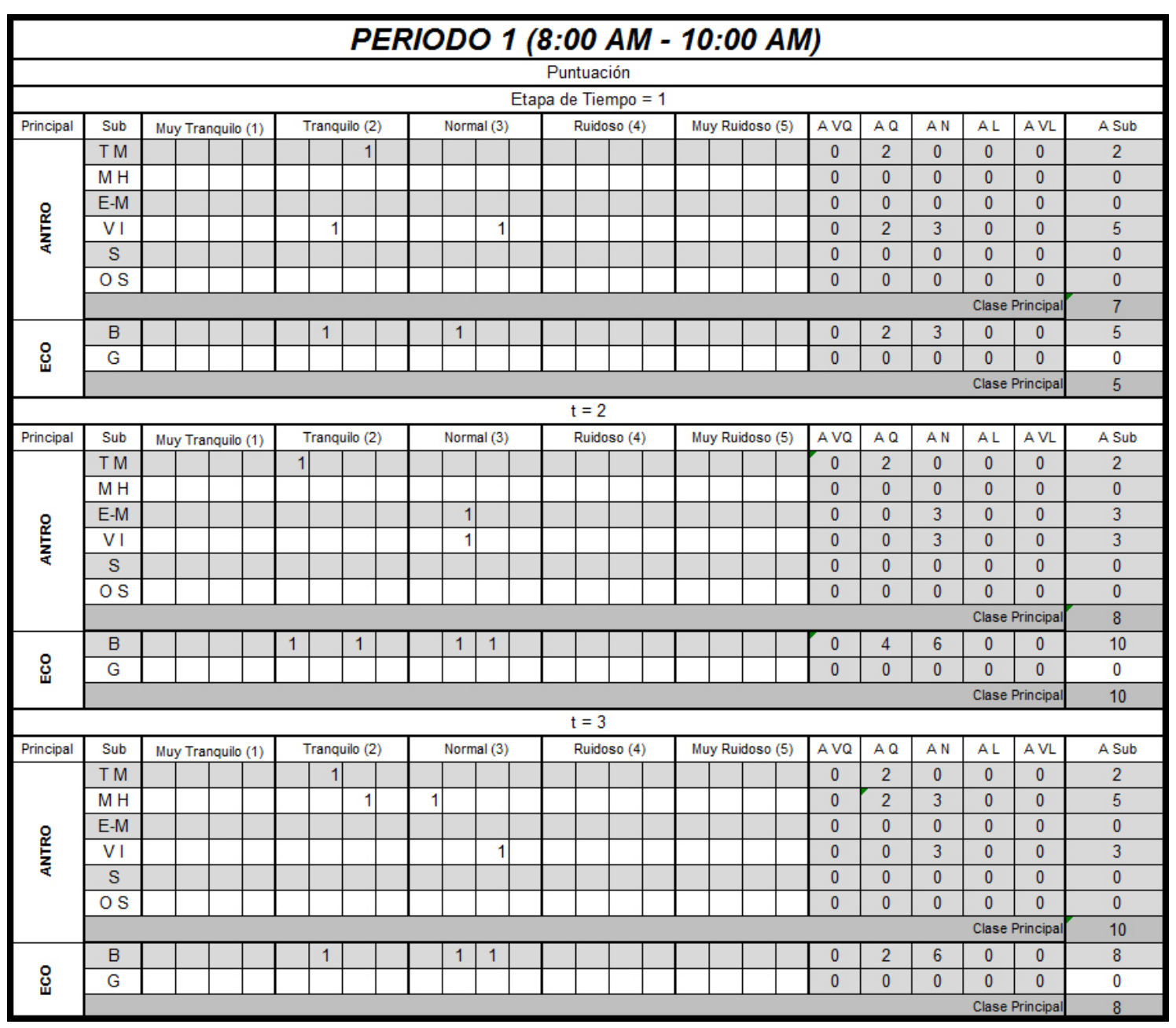

Figura 6: Formato de recolección de información para la puntuación de sonoridad. Elaboración propia.

La sonoridad total del paisaje sonoro en un sitio y periodo dado se calculó sumando las puntuaciones obtenidas de las veinte etapas de tiempo (30 s) en las cuales fue dividido cada período (10 min). Del mismo modo, la sonoridad percibida de cada sonido de clase principal del paisaje sonoro fue el resultado de la suma de las puntuaciones de su respectiva subclase, ver tabla 1. 


\begin{tabular}{|c|c|c|c|}
\hline \multirow{12}{*}{ Punto 07} & Clase Principal & Subclase & Tota \\
\hline & \multirow{7}{*}{ Antropofonía } & Transporte motorizado & 622 \\
\hline & & Movimiento humano & 294 \\
\hline & & Electromecánicos & 0 \\
\hline & & Voces e instrumentos & 488 \\
\hline & & Sociales & 96 \\
\hline & & Otros sonidos & 18 \\
\hline & & Total Clase & 1518 \\
\hline & \multirow{4}{*}{ Ecotopofonía } & Subclase & Tota \\
\hline & & Biofonía & 48 \\
\hline & & Geofonía & 17 \\
\hline & & Total Clase & 65 \\
\hline
\end{tabular}

Tabla 1: Ejemplo del acumulado de la sonoridad percibida en un punto de muestreo. Fuente: Elaboración propia.

\subsection{Análisis geoestadístico}

El procesamiento de los parámetros acústicos y perceptuales del paisaje sonoro se llevó a cabo en el programa informático $\mathrm{R}$ Studio, ajustando los diferentes modelos del variograma experimental al modelo teórico de mayor similitud. La teoría de la regionalización de las variables que constituye las bases de la predicción de datos georreferenciados respaldó este análisis (Harman, Koseoglu y Yigit, 2016). A continuación se describe la aplicación del proceso a uno de los indicadores acústicos del ruido (LEQ) y a una de las clases principales del paisaje sonoro (antropofonía).

Inicialmente se realizó un análisis exploratorio de las variables mediante el cual se determinó el carácter estacionario de los datos y se validó la aplicación del método geoestadístico. Posteriormente se seleccionó el Kriging como método de interpolación para la estimación de la información faltante, ver figura 7. 

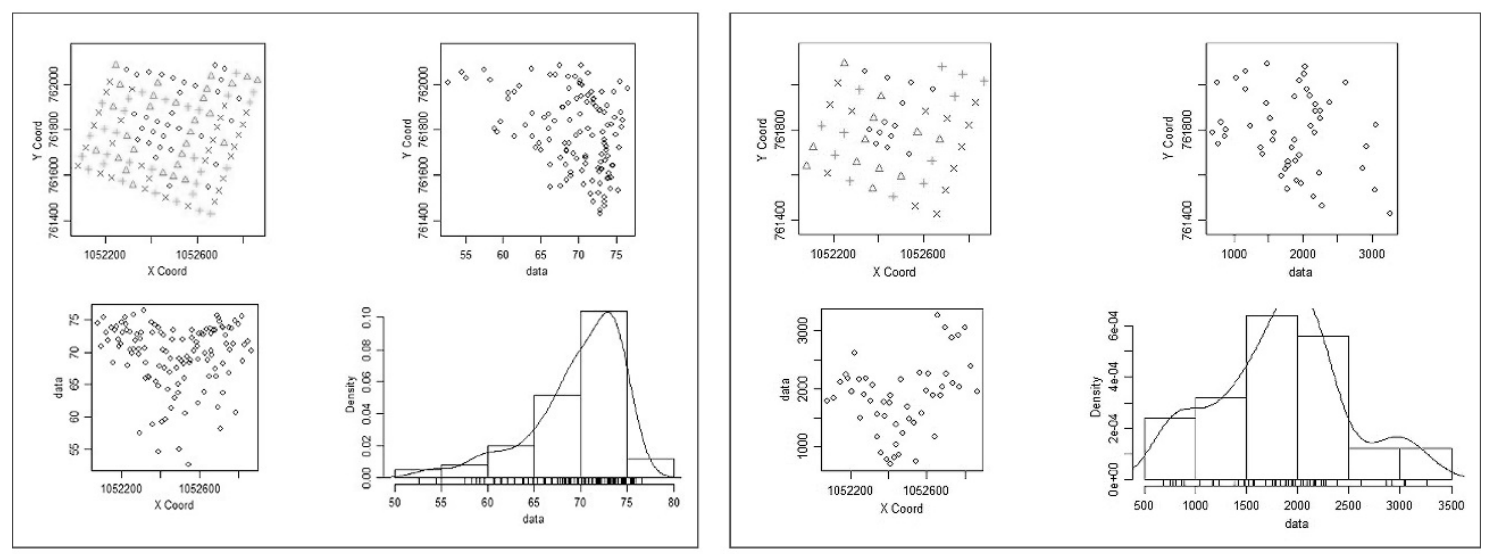

Figura 7: Análisis exploratorio del paisaje sonoro. A la izquierda los resultados del indicador acústico dBLeq y a la derecha los datos de antropofonía. Elaboración propia en R Studio.

El Kriging es un método basado en la auto correlación espacial de las variables y puede expresarse como la suma de tres componentes; el componente estructural que consiste en una media constante, un componente aleatorio, pero espacialmente correlacionado y por último el término de error residual no correlacionado espacialmente. El método utiliza un patrón de pesos, en donde los puntos más cercanos tienen mayor peso para la estimación del punto faltante (Harman et al., 2016).

La ecuación general del Kriging es:

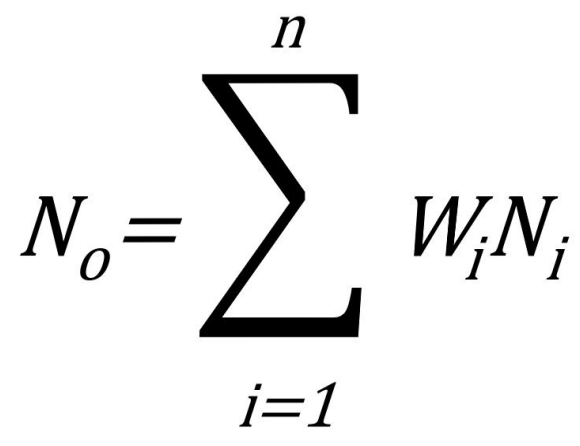

En la cual No representa el valor de ruido para interpolación del punto en $\left(x_{0}, Y_{0}\right), W_{l_{\mathrm{r}}}$ epresenta los pesos que corresponden a cada $N_{I}$ en $(X I, Y I)$ usados en el cálculo de $N o, N I$ corresponde a los valores de ruido de los puntos de referencia usados en el cálculo y finalmente $n$ representa el número de variables utilizadas en la interpolación.

Una vez definido el método de interpolación, se procede a generar los variogramas experimentales del indicador acústico Leq y de la puntuación de antropofonía (distancia máxima: $\mathrm{dBLEQ}=500$, antropofonías = 600; semivariancia: $\mathrm{dBLEQ}=20$, antropofonías $=20$ ) Así, los pesos como función directa del modelo de variograma fueron determinados para asegurar la imparcialidad en la estimación de los datos faltantes. El proceso es clave para obtener una buena interpolación, ver figura 8. 

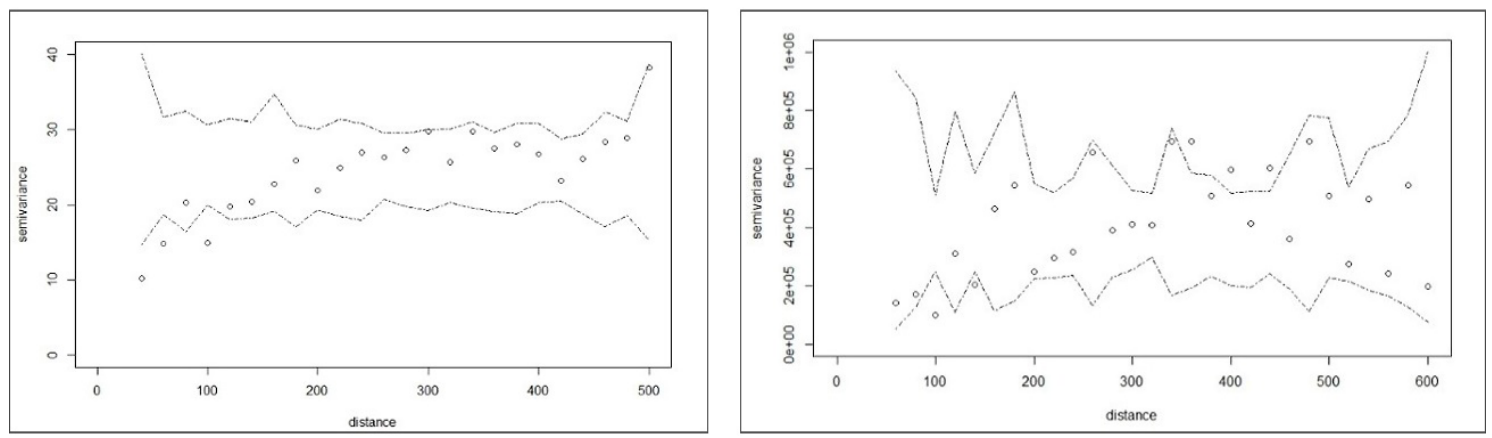

Figura 8: Variogramas experimentales del paisaje sonoro. A la izquierda los resultados del indicador acústico dBLeq y a la derecha los datos de la antropofonía. Elaboración propia en R Studio.

A partir de los variogramas experimentales se ajustó cada modelo experimental al respectivo modelo teórico según el error mínimo de estimación obtenido, los modelos teóricos utilizados fueron los siguientes; exponencial, gaussiano y esférico. Se encontró que el modelo teórico con menor probabilidad de error para la interpolación del indicador acústico LEQ fue el exponencial, mientras que para la puntuación de antropofonía fue el gaussiano, ver la figura 9.
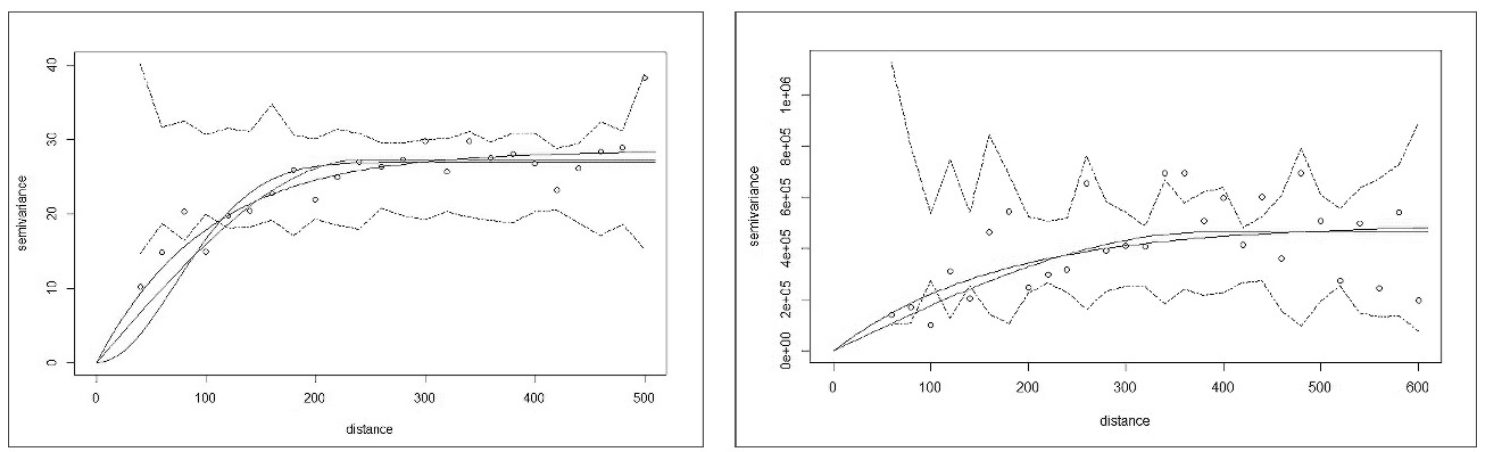

Figura 9: Ajustes del modelo experimental al modelo teórico. A la izquierda los resultados del indicador acústico dBLeq y a la derecha los datos de la antropofonía. Elaboración propia en $\mathrm{R}$ Studio.

Posteriormente se crearon las superficies de interpolación aplicando los modelos teóricos de menor índice de error. La figura 10 muestra zonas concretas del área de estudio en la que se presentaron niveles diferenciados del indicador acústico LEQ y de la sonoridad percibida de antropofonía. Se determinó así que las superficies generadas fueron congruentes y podían ser exportadas para generar los respectivos mapas. 

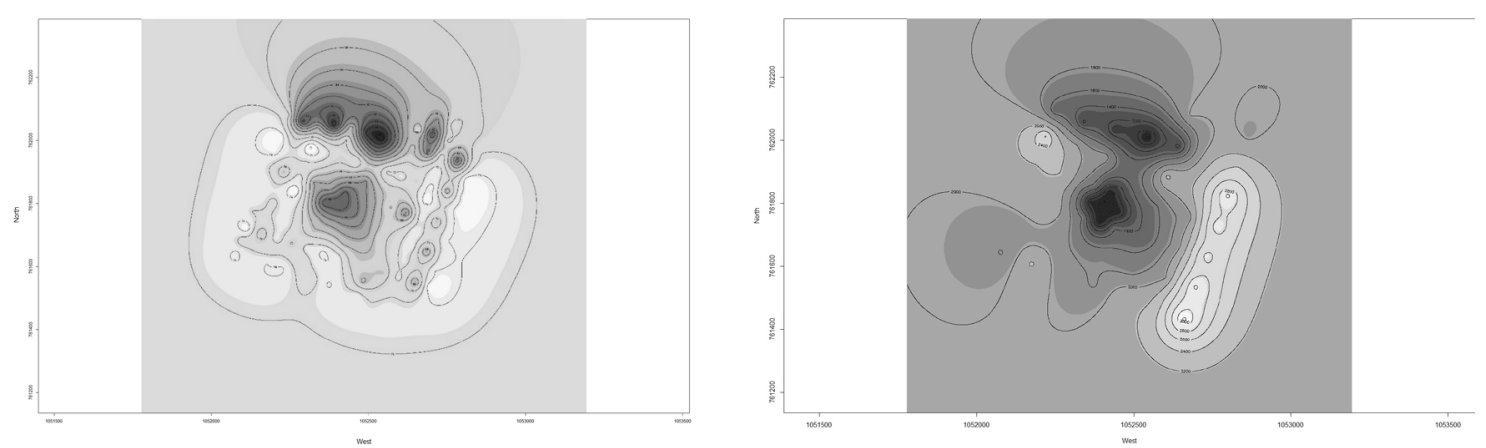

Figura 10: Superficies de interpolación del nivel de presión sonora continuo equivalente (izquierda) y de la puntuación de antropofonía (derecha). Elaboración propia en R Studio.

Cabe resaltar que el mismo análisis geo-estadístico expuesto anteriormente se realizó con los niveles mínimos dBMin y máximos dBMAX de ruido y con todas las clases que componen el paisaje sonoro urbano. Las superficies de interpolación generadas para estos datos se pueden ver en la figura 11.
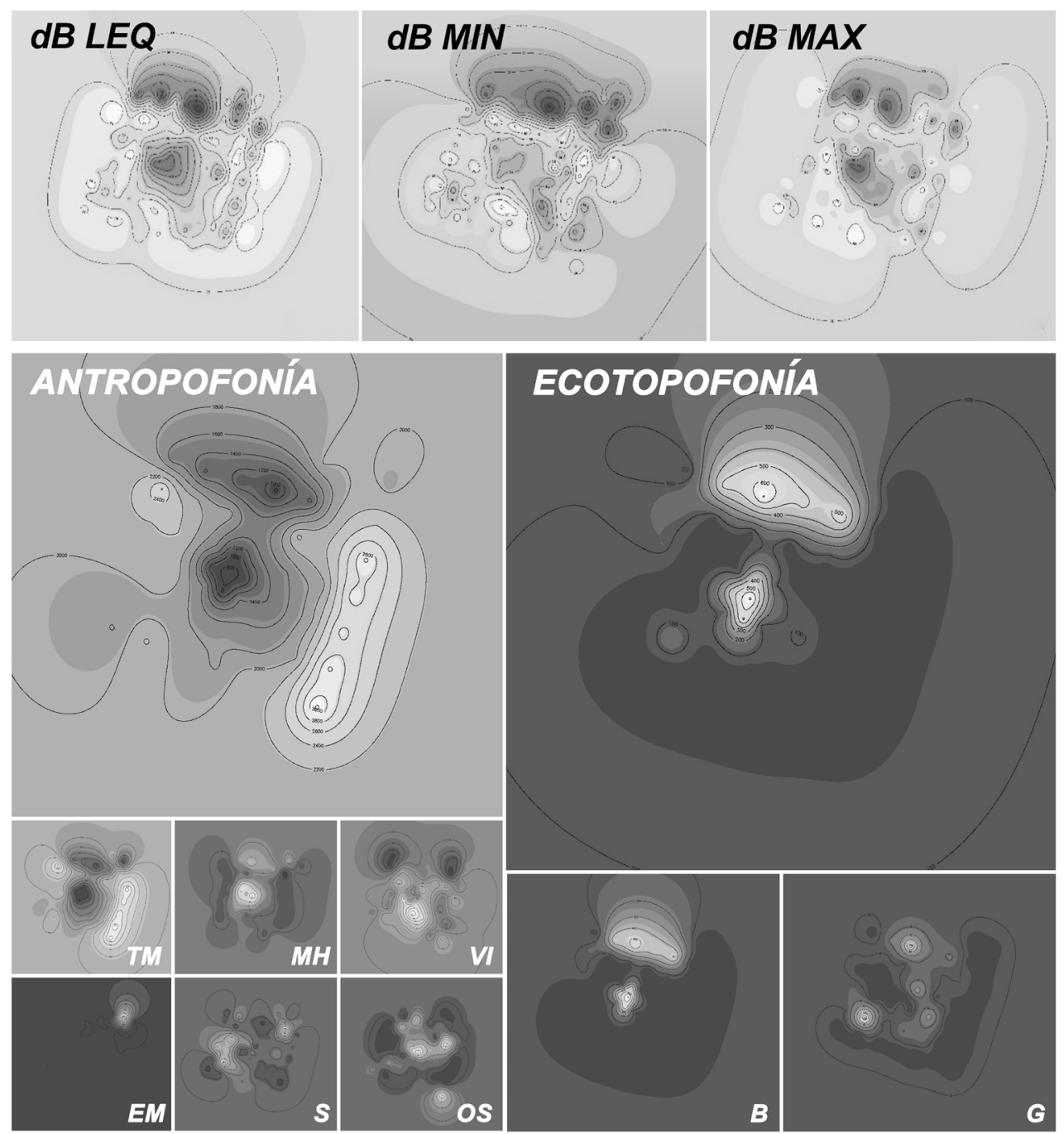

Figura 11: Superficies de interpolación de los indicadores acústicos (dBLeq, dBMin, dBMax) y de las clases principales del paisaje sonoro de con sus respectivas subclases; transporte motorizado (TM), movimiento humano (MH), voces e instrumentos (VI), electro mecánicos $(E M)$, sociales (S), Otros Sonidos (OS), biofonía (B) y geofonía (G). Elaboración propia en R Studio. 
Asimismo, superficies de interpolación también fueron realizadas para los cinco periodos de muestra de cada clase principal del paisaje sonoro urbano, con el fin de evaluar los principales cambios del comportamiento del paisaje sonoro a nivel temporal, ver figura 12.

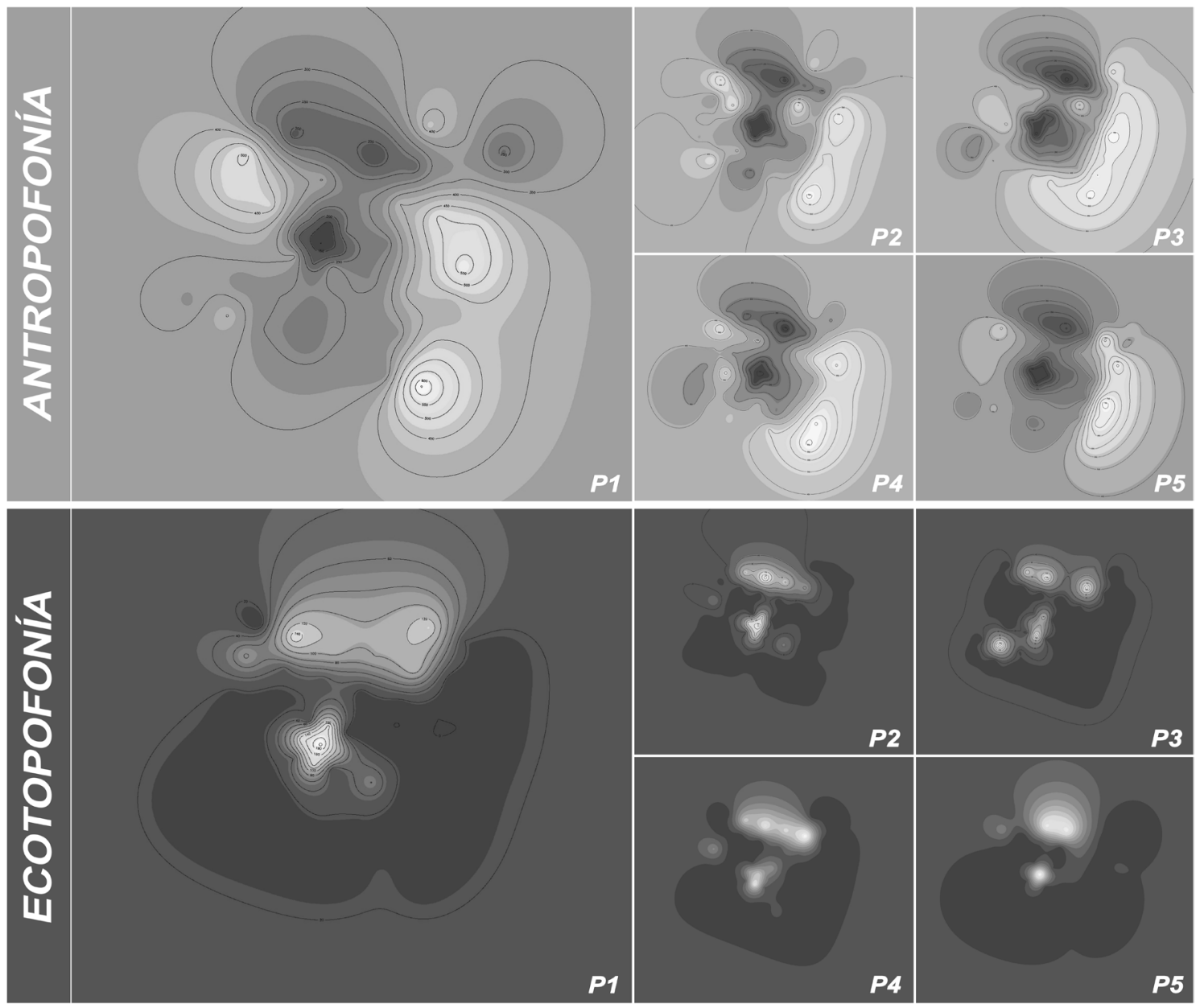

Figura 12: Superficies de interpolación para cada periodo de muestreo de sonoridad percibida. Elaboración propia en R Studio.

El análisis demostró las diferencias de los modelos teóricos que mejor se ajustaron a los modelos del variograma experimental, tanto para los indicadores acústicos como para la sonoridad percibida del paisaje sonoro. Los respectivos valores de pérdidas obtenidos para todos los datos estudiados se presentan en la tabla 2.

\begin{tabular}{|c|c|c|c|}
\hline \multirow{2}{*}{$\begin{array}{l}\text { NIVEL DE PRESIÓN } \\
\text { SONOR }\end{array}$} & \multicolumn{3}{|c|}{ Valor de pérdida } \\
\hline & Exponencial & Gaussiano & \\
\hline dBLEQ & 42,787 & 58,660 & 50,202 \\
\hline $\mathrm{dBM}$ & 63,096 & 92,980 & 82,816 \\
\hline $\mathrm{dBM}_{\mathrm{Ax}}$ & 561,227 & 444,527 & 318,792 \\
\hline
\end{tabular}




\begin{tabular}{|c|c|c|c|c|}
\hline \multirow{3}{*}{\multicolumn{2}{|c|}{$\begin{array}{l}\text { PUNTUACIÓN DEL } \\
\text { PAISAJE SONORO }\end{array}$}} & \multicolumn{3}{|c|}{ Valor de pérdida } \\
\hline & & & & \\
\hline & & Exponencial & Gaussiano & \\
\hline \multicolumn{2}{|c|}{ Antropofonías } & $53,824,508,019,656$ & $53,217,540,117,794$ & $54,000,797,898,791$ \\
\hline \multirow[b]{6}{*}{ 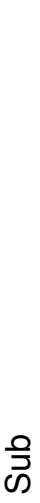 } & T.M. & $22,878,549,540,707$ & $21,882,585,745,903$ & $22,942,722,897,659$ \\
\hline & M.H. & $15,552,742,356$ & $16,911,438,545$ & $22,338,281,558$ \\
\hline & E.M. & $209,428,802,505$ & $209,451,361,161$ & $209,127,941,062$ \\
\hline & V.I. & $33,503,519,722$ & $54,085,874,047$ & $83,133,826,636$ \\
\hline & S. & $270,146,463$ & $254,957,024$ & $255,761,617$ \\
\hline & O.S. & 594,590 & 665,375 & 686,873 \\
\hline \multicolumn{2}{|c|}{ Ecotopofonías } & $2,975,070,825,483$ & $3,559,920,262,382$ & $4,196,720,218,684$ \\
\hline \multirow[b]{2}{*}{$\stackrel{\circ}{\vec{D}}$} & B. & $3,491,155,083,042$ & $4,120,921,302,636$ & $4,788,739,633,250$ \\
\hline & G. & $49,477,730$ & $50,282,028$ & $51,196,399$ \\
\hline
\end{tabular}

Tabla 2: Valores de pérdida de los datos analizados. Fuente: Elaboración propia.

El análisis geoestadístico descrito en este nivel sugiere que de los métodos más utilizados para la interpolación de variables espaciales, como el PDI (Pesos de la Distancia Inversa), el Multicuadrático y el Kriging, este último es de las mejores técnicas de aplicación en la predicción de datos sonoros a nivel territorial. (Harman et al., 2016).

\subsection{Mapeo del ruido}

Mediciones de los niveles equivalentes, niveles mínimos y niveles máximos de presión sonora se tomaron en todos los puntos dispuestos del área de estudio. De esta forma la distribución de ruido fue calculada mediante el método de interpolación expuesto anteriormente y se elaboraron cartografías para cada indicador acústico (LEQ, LMIN, LMAX), utilizando ArcMap 10.3.1.

Asimismo criterios de sistematización y comprensión del sonido se tuvieron en cuenta para ajustar los esquemas de color de los mapas. Se aplicó una secuencia de tonos verdes y amarillos brillantes pasando por naranjas hasta llegar al rojo oscuro, de esta forma los valores bajos se obtuvieron en tonos verdes mientras que los valores cercanos a los $65 \mathrm{~dB}$ estuvieron representados en rojo, evidenciando así el potencial peligro de exposición al ruido urbano (Schiewe y Weninger, 2013), ver figura 13. 


\begin{tabular}{|c|c|c|c|}
\hline & сMYK & $R G B$ & Hexadecimal \\
\hline \multicolumn{4}{|c|}{$\begin{array}{l}\text { Niveles de presion sonora } \\
\text { Clases de acuerdo a Alemania DIN 180005-2 }\end{array}$} \\
\hline$<=35$ & $\mathrm{C}=7 \mathrm{M}=0 \mathrm{Y}=37 \mathrm{~K}=0$ & $R=244 \mathrm{G}=242 \mathrm{~B}=184$ & \#F4F2B8 \\
\hline$>35-40$ & $\mathrm{C}=7 \mathrm{M}=0 \mathrm{Y}=52 \mathrm{~K}=0$ & $R=245 G=239 B=150$ & \#F5EF96 \\
\hline$>40-45$ & $C=13 \mathrm{M}=0 \mathrm{Y}=65 \mathrm{~K}=0$ & $R=235 \mathrm{G}=231 \mathrm{~B}=117$ & \#EBE775 \\
\hline$>45-50$ & $C=10 M=0 \quad Y=87 \quad K=0$ & $R=242 \mathrm{G}=230 \mathrm{~B}=43$ & \#F9CB1D \\
\hline$>50-55$ & $C=3 \mathrm{M}=20 \mathrm{Y}=90 \mathrm{~K}=0$ & $R=249 G=203 \quad B=29$ & \#F7AD1A \\
\hline$>55-60$ & $\mathrm{C}=0 \mathrm{M}=38 \mathrm{Y}=92 \mathrm{~K}=0$ & $R=247 \mathrm{G}=173 \mathrm{~B}=26$ & \#F7AD1A \\
\hline$>60-65$ & $C=0 M=59 \quad Y=92 \quad K=0$ & $R=239 \mathrm{G}=130 \mathrm{~B}=34$ & \#EF8222 \\
\hline$>65-70$ & $C=0 M=87 Y=95 K=0$ & $R=229 \mathrm{G}=62 \mathrm{~B}=31$ & \#E53E1F \\
\hline$>70-75$ & $C=10 M=100 Y=98 K=2$ & $R=209 \mathrm{G}=2 \mathrm{~B}=31$ & \#D1021F \\
\hline$>75-80$ & $\mathrm{C}=24 \mathrm{M}=100 \mathrm{Y}=99 \mathrm{~K}=22$ & $R=160 \mathrm{G}=20 \mathrm{~B}=29$ & \#A0141D \\
\hline$>80$ & $\mathrm{C}=52 \mathrm{M}=100 \mathrm{Y}=99 \mathrm{~K}=22$ & $R=120 \mathrm{G}=31 \mathrm{~B}=32$ & \#781F20 \\
\hline \multicolumn{4}{|c|}{$\begin{array}{l}\text { Niveles de presión sonora dB Min (A) } \\
\text { Construcción de clases a partir de la información del estudio }\end{array}$} \\
\hline$<=49$ & $C=7 \mathrm{M}=0 \mathrm{Y}=37 \mathrm{~K}=0$ & $R=244 G=242 B=184$ & \#F4F2B8 \\
\hline$>50-52$ & $C=7 M=0 Y=52 K=0$ & $R=245 G=239 B=150$ & \#F5EF96 \\
\hline$>53-55$ & $C=13 \mathrm{M}=0 \mathrm{Y}=65 \mathrm{~K}=0$ & $R=235 \mathrm{G}=231 \mathrm{~B}=117$ & \#EBE775 \\
\hline$>56-57$ & $C=10 M=0 \quad Y=87 \mathrm{~K}=0$ & $\mathrm{R}=242 \mathrm{G}=230 \mathrm{~B}=43$ & \#F9CB1D \\
\hline$>58-60$ & $C=3 \mathrm{M}=20 \mathrm{Y}=90 \mathrm{~K}=0$ & $R=249 \mathrm{G}=203 \mathrm{~B}=29$ & \#F7AD1A \\
\hline$>61-62$ & $C=0 M=38 \quad Y=92 K=0$ & $R=247 \mathrm{G}=173 \mathrm{~B}=26$ & \#F7AD1A \\
\hline$>69$ & $C=0 M=66 \quad Y=85 K=0$ & $R=244 G=132 B=46$ & \#F4842E \\
\hline \multicolumn{4}{|c|}{$\begin{array}{l}\text { Niveles de presión sonora dB Leq (A) } \\
\text { Construcción de clases a partir de la información del estudio }\end{array}$} \\
\hline$<=64$ & $C=10 M=0 \quad Y=87 \mathrm{~K}=0$ & $R=242 G=230 B=43$ & \#F9CB1D \\
\hline$>65-67$ & $C=3 \quad M=20 \quad Y=90 \quad K=0$ & $R=249 \mathrm{G}=203 \mathrm{~B}=29$ & \#F7AD1A \\
\hline$>68-69$ & $C=0 \quad M=38 \quad Y=92 \quad K=0$ & $R=247 \mathrm{G}=173 \mathrm{~B}=26$ & \#F7AD1A \\
\hline$>70-72$ & $C=0 M=87 \quad Y=95 \quad K=0$ & $R=229 \mathrm{G}=62 \mathrm{~B}=31$ & \#E53E1F \\
\hline$>73-75$ & $C=10 M=100 \quad Y=98 K=2$ & $R=209 \mathrm{G}=2 \mathrm{~B}=31$ & \#D1021F \\
\hline$>76$ & $C=24 M=100 Y=99 \mathrm{~K}=22$ & $R=160 \mathrm{G}=20 \mathrm{~B}=29$ & \#A0141D \\
\hline \multicolumn{4}{|c|}{$\begin{array}{l}\text { Niveles de presión sonora dB Max (A) } \\
\text { Construcción de clases a partir de la información del estudio }\end{array}$} \\
\hline$<=83$ & $C=0 M=38 \quad Y=92 \quad K=0$ & $R=247 \mathrm{G}=173 \mathrm{~B}=26$ & \#F7AD1A \\
\hline$>84-86$ & $\mathrm{C}=0 \mathrm{M}=59 \mathrm{Y}=92 \mathrm{~K}=0$ & $R=239 \mathrm{G}=130 \mathrm{~B}=34$ & \#EF8222 \\
\hline$>87-88$ & $C=0 M=87 \quad Y=95 \quad K=0$ & $R=229 \mathrm{G}=62 \mathrm{~B}=31$ & \#E53E1F \\
\hline$>89-91$ & $C=10 M=100 Y=98 \mathrm{~K}=2$ & $R=209 \mathrm{G}=2 \mathrm{~B}=31$ & \#D1021F \\
\hline$>92-94$ & $C=24 M=100 \mathrm{Y}=99 \mathrm{~K}=22$ & $R=160 \mathrm{G}=20 \mathrm{~B}=29$ & \#A0141D \\
\hline$>95-96$ & $C=52 M=100 Y=99 \mathrm{~K}=22$ & $R=120 \mathrm{G}=31 \mathrm{~B}=32$ & \#781F20 \\
\hline$>97$ & $\mathrm{C}=34 \mathrm{M}=100 \mathrm{Y}=73 \mathrm{~K}=75$ & $R=71 \mathrm{G}=10 \mathrm{~B}=22$ & $\# 470$ A16 \\
\hline
\end{tabular}

Figura 13: Ajustes de los esquemas de colores para el mapeo del ruido. Elaboración propia. Mapeo del paisaje sonoro

El mapeo del paisaje sonoro urbano es básicamente la visualización del patrón de comportamiento del paisaje sonoro a escala temporal y espacial. Este estudio elaboró mapas para cada clase principal y sus respectivas subclases de sonido, teniendo como base la sonoridad total acumulada por periodo en cada punto 
de muestreo. Por consiguiente, la variación del comportamiento espaciotemporal del paisaje sonoro urbano pudo ser representada.

Además, superficies de interpolación generadas en R Studio para cada variable del paisaje sonoro urbano fueron procesadas en el sistema de información geográfica ArcMap 10.3.1, ajustando los correspondientes rangos de las puntuaciones de sonoridad y asegurando el mapeo final del paisaje sonoro urbano en todos sus niveles taxonómicos.

\subsection{Comparación de parámetros físicos y perceptuales del sonido}

La comparación entre las cartografías sonoras obtenidas en este estudio fue realizada, integrando tanto el mapeo de las puntuaciones del paisaje sonoro en donde se evaluó la percepción de variabilidad espaciotemporal del entorno acústico urbano como el mapeo del ruido urbano que expreso los valores en $\mathrm{dB}(\mathrm{A})$ de los indicadores acústicos del sonido. La información puso en contraste los valores objetivos y subjetivos del paisaje sonoro, como determinante en la afectación de la experiencia sonora de la ciudad.

\section{Cartografías sonoras}

En esta sección se revisan los mapas obtenidos a partir del estudio integral del paisaje sonoro urbano, ligando el sonido de la ciudad desde el nivel físico-perceptual y su consecuente potencial como instrumento de ordenamiento del territorio.

\section{1 Mapas de ruido}

El mapa del nivel de presión sonora equivalente (LEQ) indicó que los niveles oscilaron entre 55 a 75 $\mathrm{dBA}$, ver figura 14. El comportamiento espacial de este parámetro tuvo relación directa con el parque principal del lugar, localizado en el centro del área de estudio, y con los parques que bordean la ronda del Rio Molino al norte de la misma área. Los parques urbanos fueron las zonas que más bajos niveles dBLEQ presentaron, con valores de entre 55 a 60 dBA mientras que las demás zonas alcanzaron niveles de 65 a 76 dBA. 


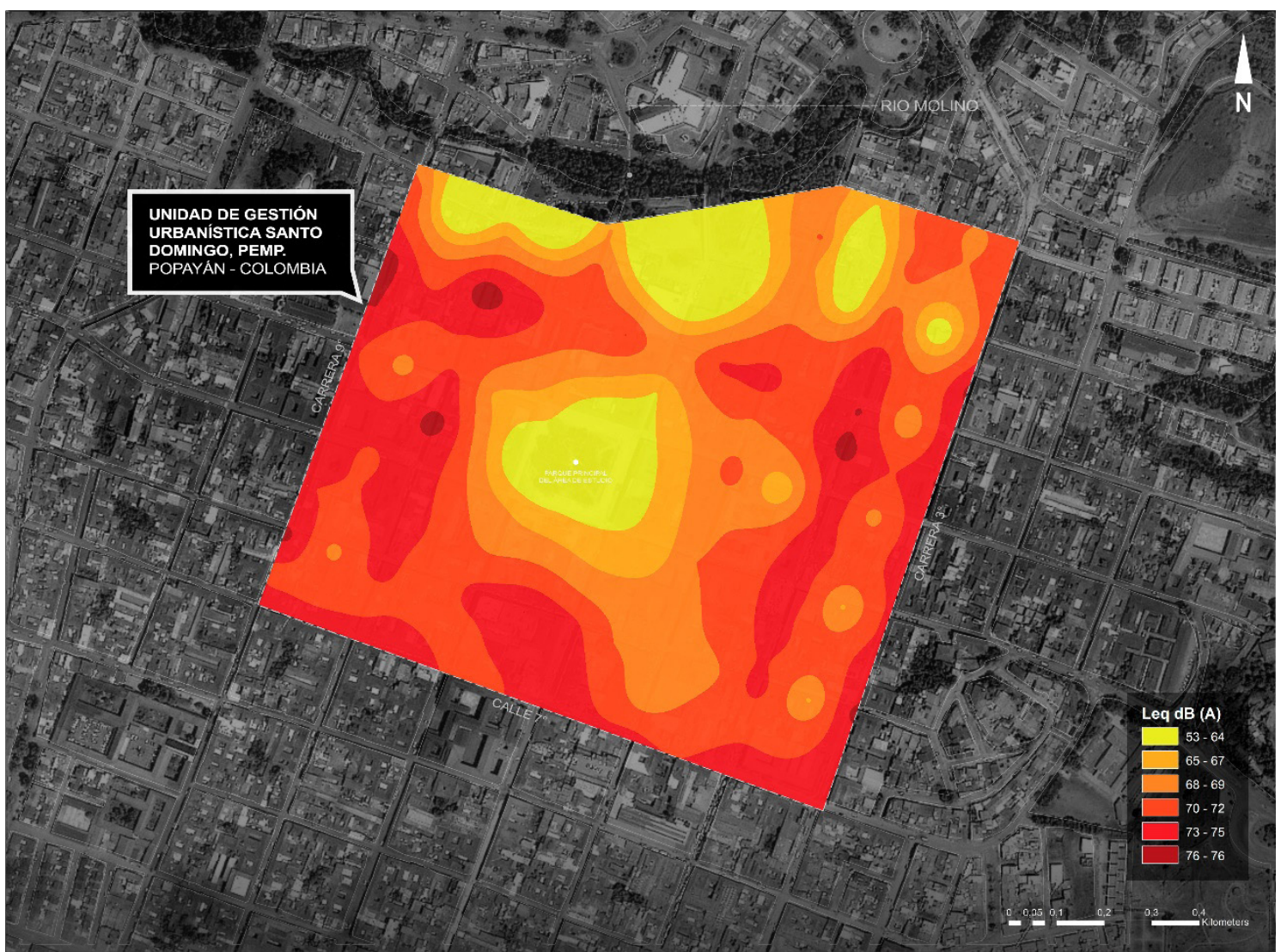

Figura 14: Mapa de ruido del nivel de presión sonora equivalente, Popayán - Colombia. Elaboración propia.

En cuanto al mapa del nivel máximo de presión sonora ( $\mathrm{Lmax}$ ) los rangos estuvieron entre 70 a $95 \mathrm{dBA}$, las zonas expuestas a niveles excesivos de ruido corresponden a los lugares con mayor intensidad de tráfico vehicular, intersecciones viales en donde aumenta la congestión del tráfico y sectores de actividad comercial no moderada. La medición más alta registro los 98 dBA, ver mapa en la figura 15. 


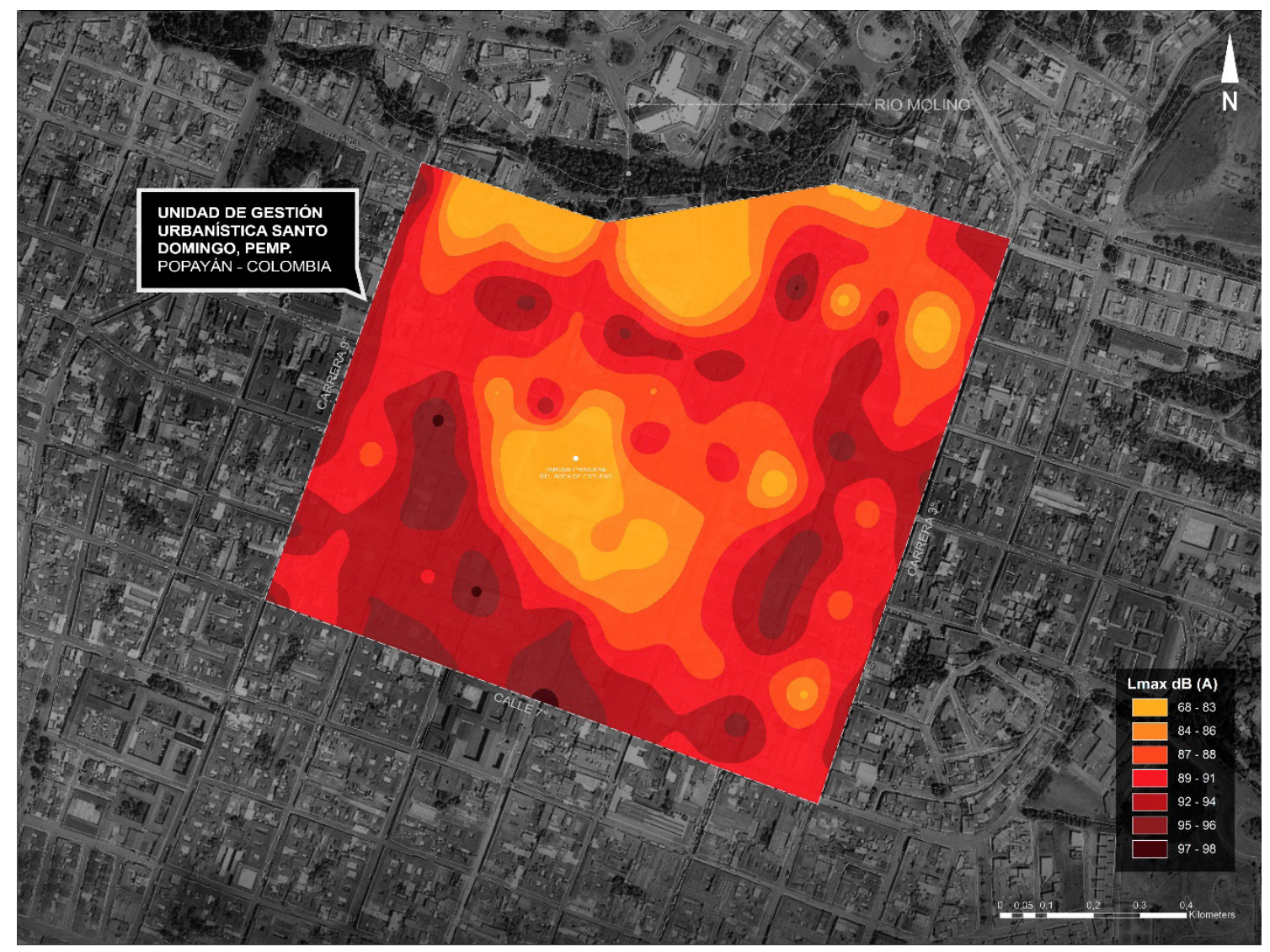

Figura 15: Mapa de ruido del nivel máximo de presión sonora, Popayán - Colombia. Elaboración propia.

Finalmente el mapa del nivel mínimo de presión sonora (Lmin) presentó un rango de variación de entre 45 y 60 dBA como se muestra en la figura 16 los niveles de menor registro se localizaron en el parque principal y sobre la ronda del Rio Molino, lógicamente fueron zonas de bajo flujo vehicular que mantuvieron niveles de entre 45 y $55 \mathrm{dBA}$. 


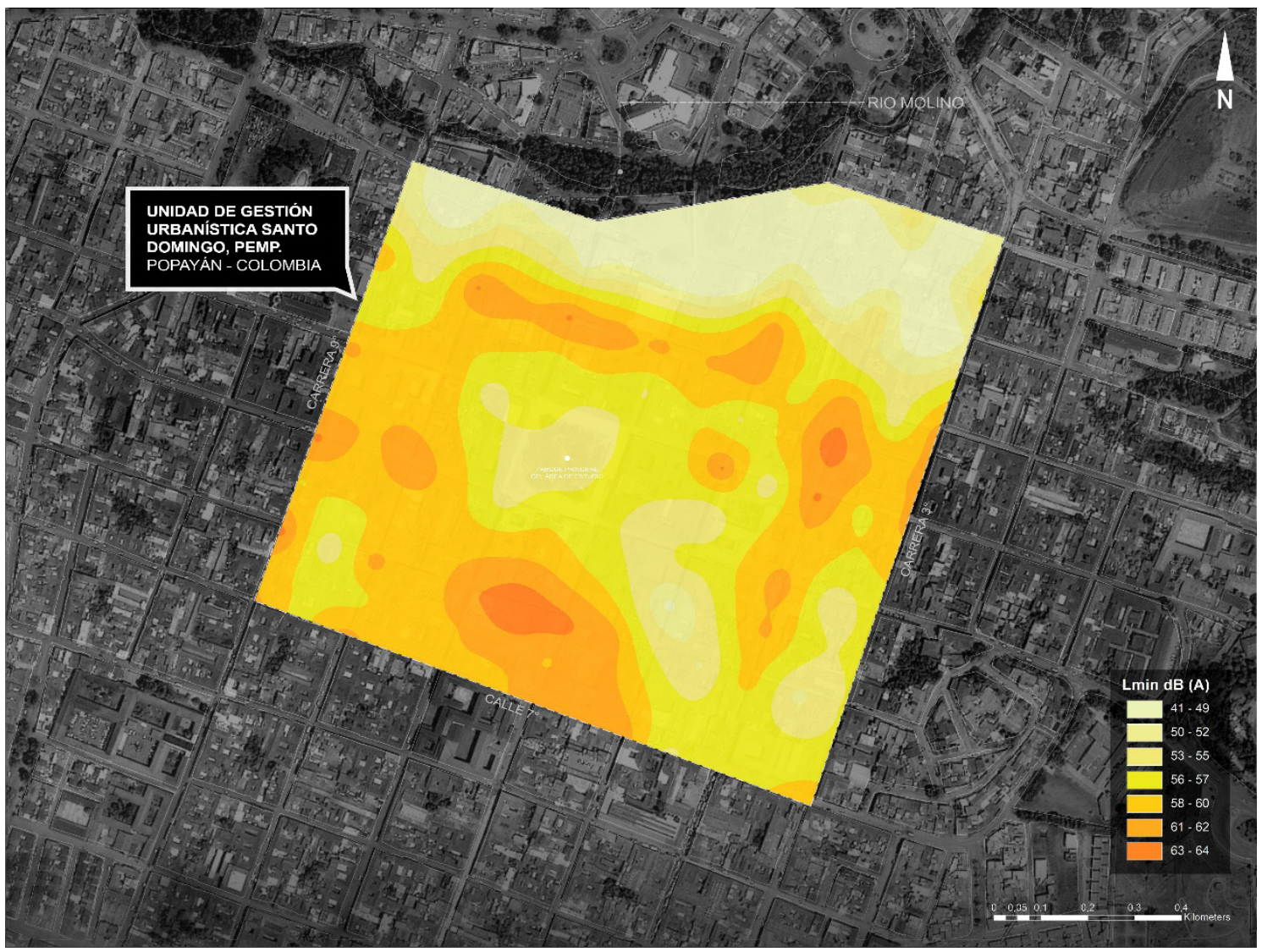

Figura 16: Mapa de ruido del nivel mínimo de presión sonora, Popayán - Colombia. Elaboración propia.

\subsection{Mapas del paisaje sonoro}

Los mapas de antropofonía y ecotopofonía que describen el comportamiento espacial del paisaje sonoro pueden observarse en la figura 17a-b. La antropofonía fue percibida en mayor grado al oriente del área de estudio como consecuencia del alto flujo vehicular, sin embargo esta clase de sonido no dejo de percibirse a través de toda el área, ver figura 17a. El gradiente del paisaje sonoro en donde los parques urbanos funcionaron como elementos ordenadores del sonido fue demostrado (Jooa, Gageb y Kastenc, 2011). Confirmando lo anterior, la figura 17b demostró la dinámica inversa de la ecotopofonía respecto a la antropofonía, la cual concentro su sonido en los parques urbanos principalmente. 

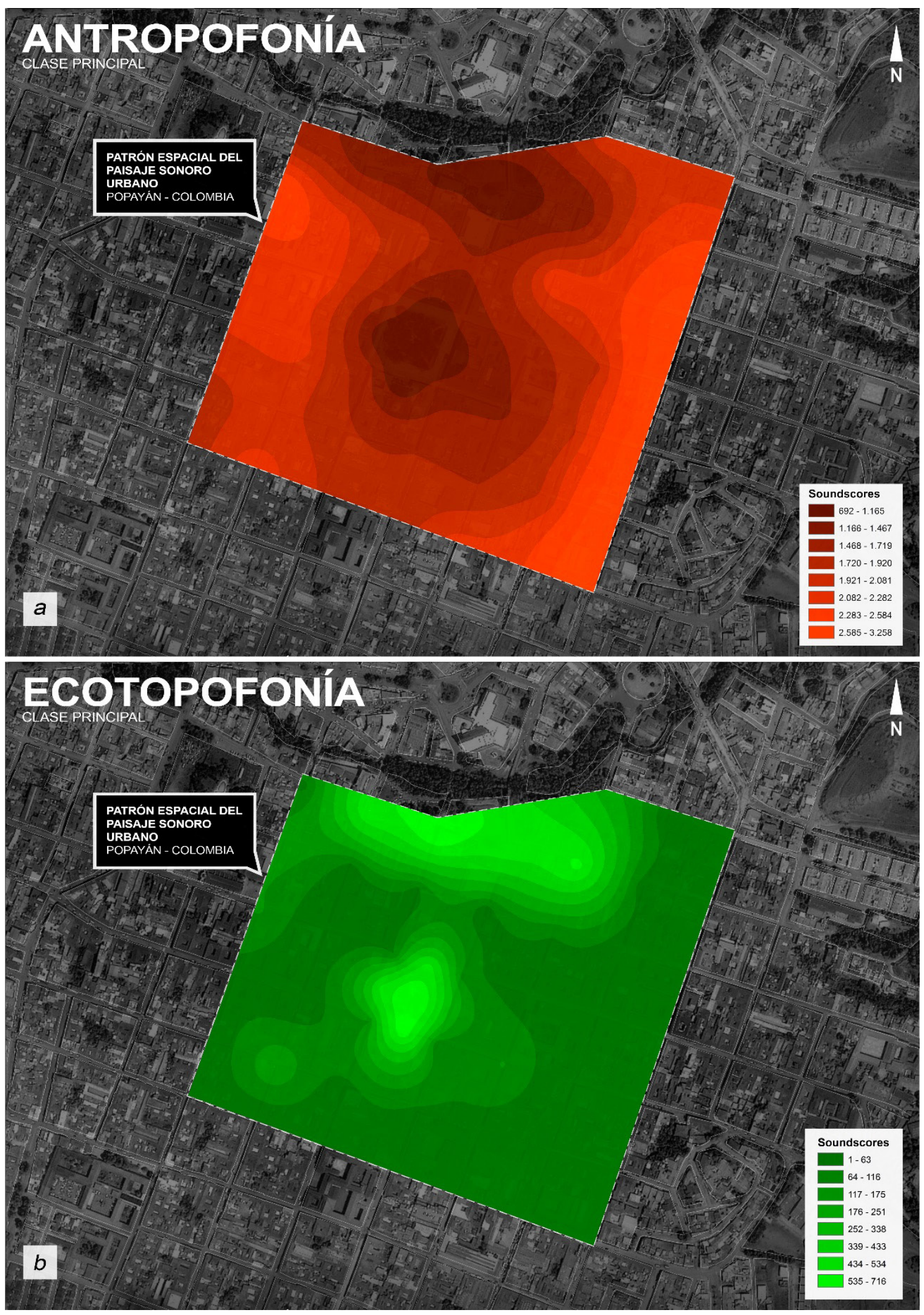

Figura 17a-b: Mapas del acumulado de la sonoridad percibida de las clases principales antropofonía (a) y ecotopofonía (b). Elaboración propia. 
La figura 18 muestra los mapas de subclase de antropofonía, el mapa de transporte motorizado (figura 18 TM) de similitud a su clase principal domino la impresión global del paisaje sonoro. Los sonidos del movimiento humano se agruparon en el parque principal del área de estudio y fueron originados por pasos de peatones en su mayoría (figura $18 \mathrm{MH}$ ), por otro lado un comportamiento diverso se reflejó en el mapa de voces e instrumentos producido por el habla, las risas y los gritos (figura $18 \mathrm{VI}$ ). Sonidos electromecánicos también se identificaron en la esquina noroccidental de su respectivo mapa (figura 18 EM) y el mapa de sonidos sociales (figura $18 \mathrm{~S}$ ) presento sonoridades acumuladas por el sonido del silbato de auxiliares de policías y sirenas de ambulancias.
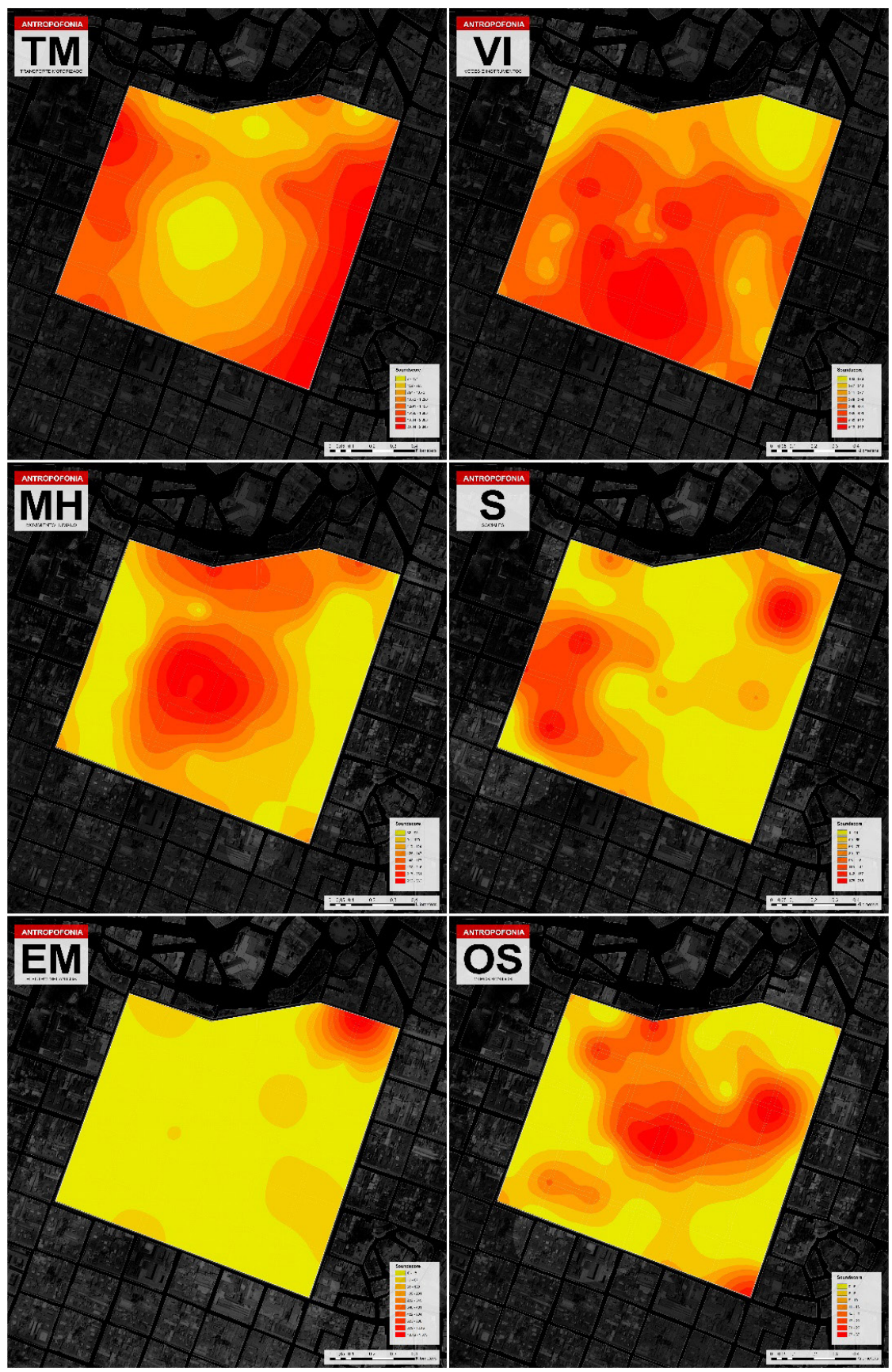

Figura 18: Acumulado de cada subclase de sonido correspondiente a la antropofonía; transporte motorizado (TM), movimiento humano $(\mathrm{MH})$, voces e instrumentos $(\mathrm{VI})$, electro mecánicos $(\mathrm{EM})$, sociales (S) y otros sonidos (OS). Elaboración propia. 
Del mismo modo los mapas de subclase de ecotopofonía se relacionan en la figura 19, el mapa de biofonía expuso un comportamiento similar al de su clase principal, en este caso el canto de las aves fue el sonido mayormente percibido (figura 19 B). Por otro lado el mapa de geofonías con un patrón de comportamiento disperso presento concentraciones de sonoridad al suroccidente y al norte del mapa (figura $19 \mathrm{G})$, sonidos generados por la lluvia y la brisa del viento fueron los más representativos.
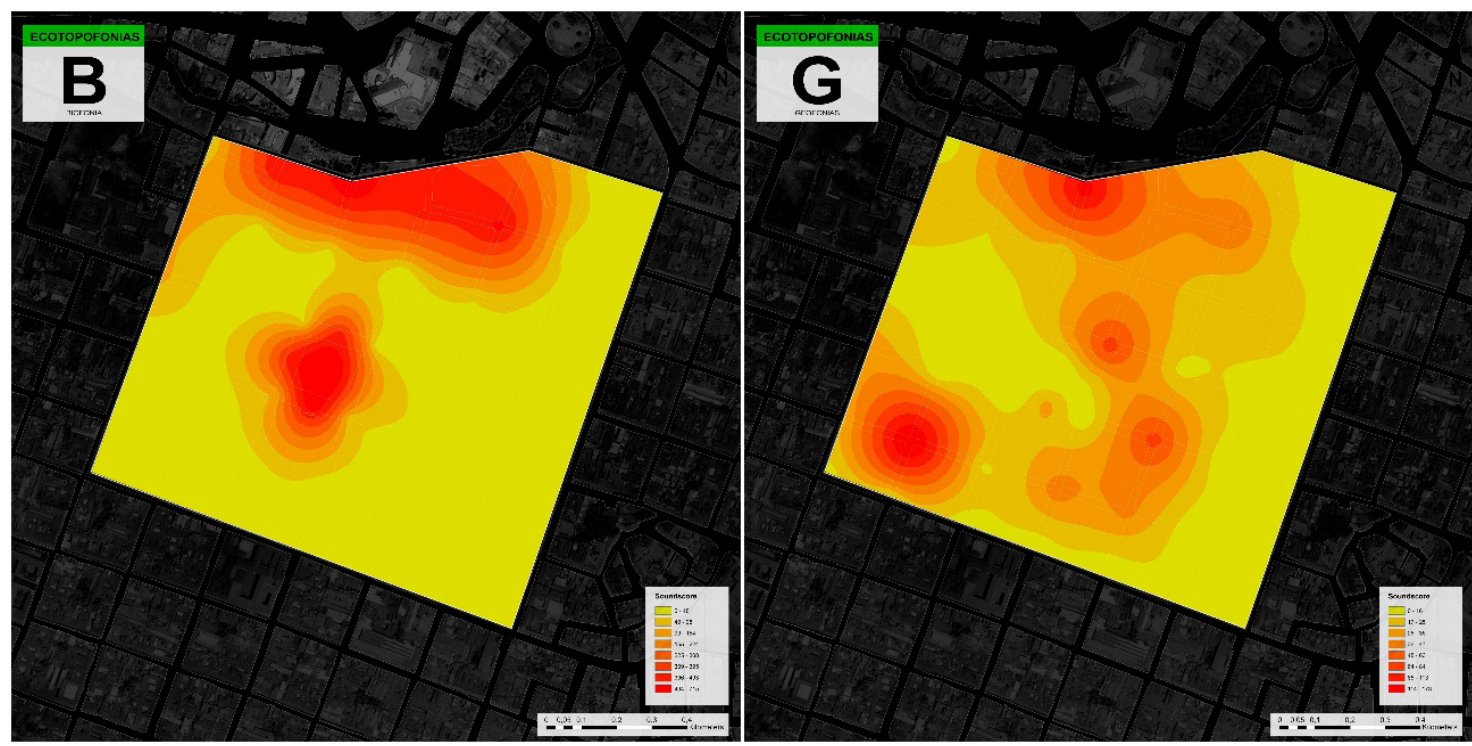

Figura 19: Acumulado de cada subclase de sonido correspondiente a la ecotopofonía; biofonías (B), geofonías $(\mathrm{G})$. Elaboración propia.

A nivel temporal los patrones de comportamiento espaciotemporal que pueden ser visualizados en los mapas de antropofonía de la figura 20 presentaron similitud entre sí, la constante de sonidos humanos durante el transcurso del día indica el continuo sonoro de la ciudad. Además la secuencia temporal confirmo un incremento sustancial en el tercer periodo de muestreo (12:00m - 2:00 pm) al oriente del área de estudio, como consecuencia de la actividad urbana. 


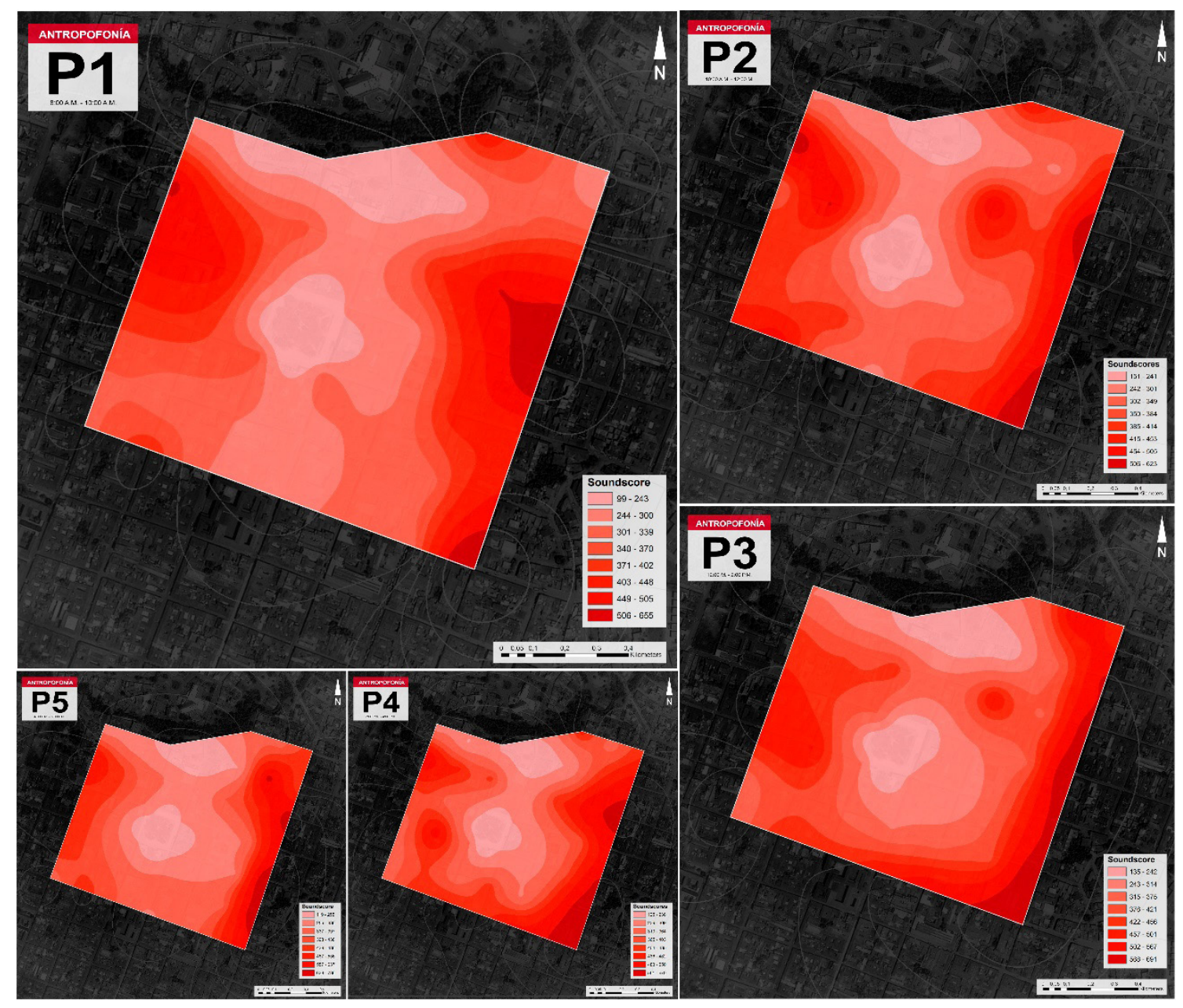

Figura 20: Patrón temporal de antropofonía. Elaboración propia.

Por otro lado la figura 21 muestra la secuencia temporal de los mapas de ecotopofonía con patrones de comportamiento agrupados al centro y nororiente de la pieza urbana, en este nivel se identificó también el continuo sonoro de esta clase de sonido. La constante de sonidos naturales producidos por la actividad del ecosistema se percibió mayormente en el primer periodo de muestreo (8:00 am - 10:00 am) y alcanzo su nivel mínimo en el quinto periodo de registro (4:00 pm - 5:00 pm). En el tercer periodo de muestreo (12:00 $m-2: 00 \mathrm{pm}$ ) se registraron geofonías al suroccidente del área de estudio que afectaron la impresión global del mapa de ecotopofonía. 


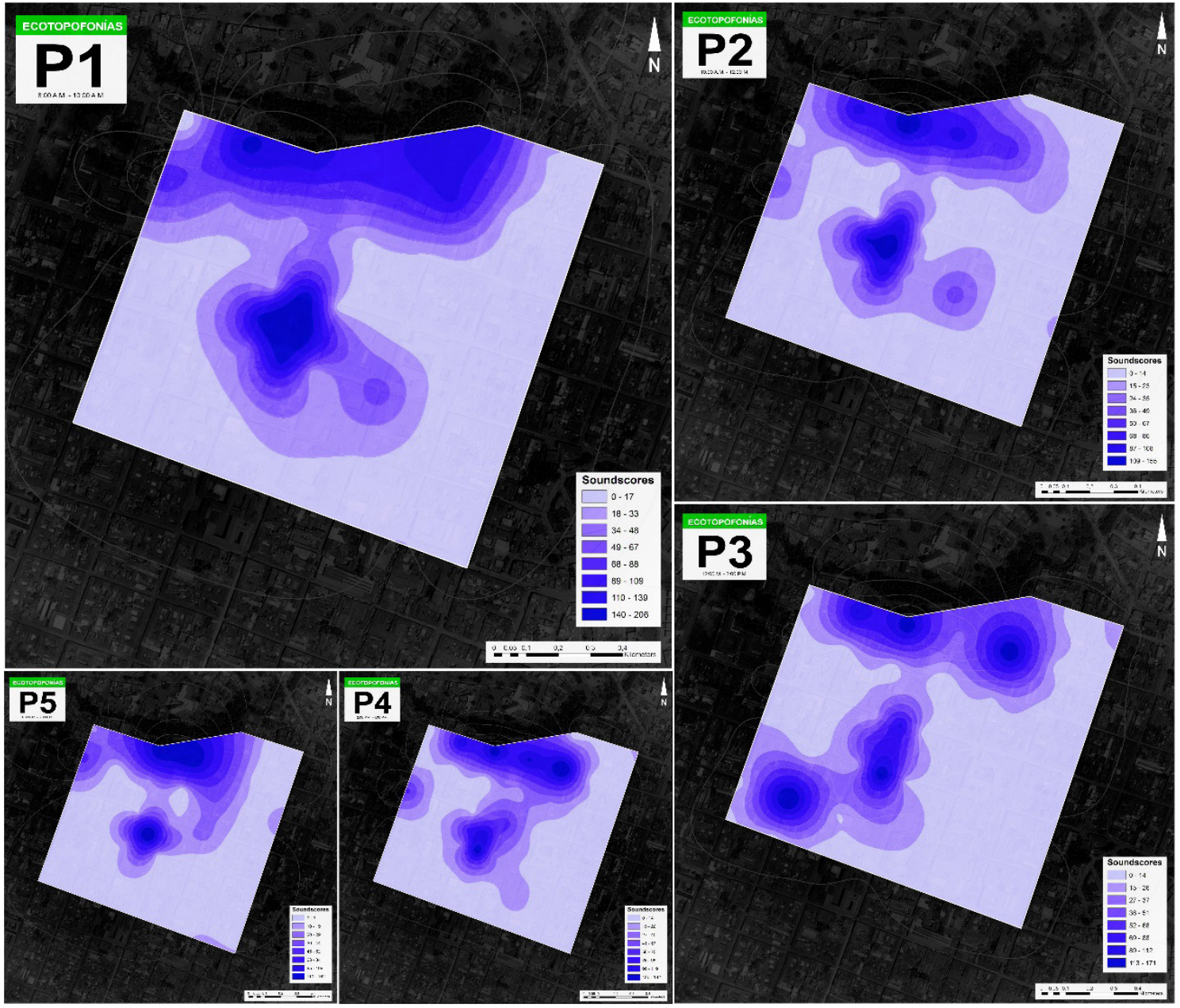

Figura 21: Patrón temporal de ecotopofonía. Elaboración propia.

Para concluir las secuencias temporales de mapas de antropofonía y ecotopofonía demostraron la correspondencia espaciotemporal entre sí. Al comparar la secuencia temporal de los mapas se encontró que los sonidos generados por la actividad humana incrementaron con el transcurrir del día y se organizan en función de entornos urbanos con mayor presencia de sonidos producidos por la naturaleza.

\subsection{Paisaje sonoro integrado}

Mediante la visualización de cartografías sonoras se integraron los indicadores acústicos del sonido y la percepción del entorno acústico urbano en un área concreta del sector histórico de Popayán. Se definió la relación entre los niveles presión sonora y los diferentes patrones de comportamiento espaciotemporal del paisaje sonoro urbano.

La figura 22 muestra que las zonas con más bajos niveles de presión sonora se caracterizaron por entornos acústicos con bajas puntuaciones de antropofonía. Situación inversa sucedió con la ecotopofonía, la cual se percibió en mayor grado cuando se registraron bajos niveles de ruido. Se infiere entonces que el paisaje sonoro con bajos niveles de presión sonora favorece la escucha del hábitat natural, facilitado así la configuración de entornos acústicos de calidad para la población y que dependen de los procesos urbanísticos 
en todos sus niveles.

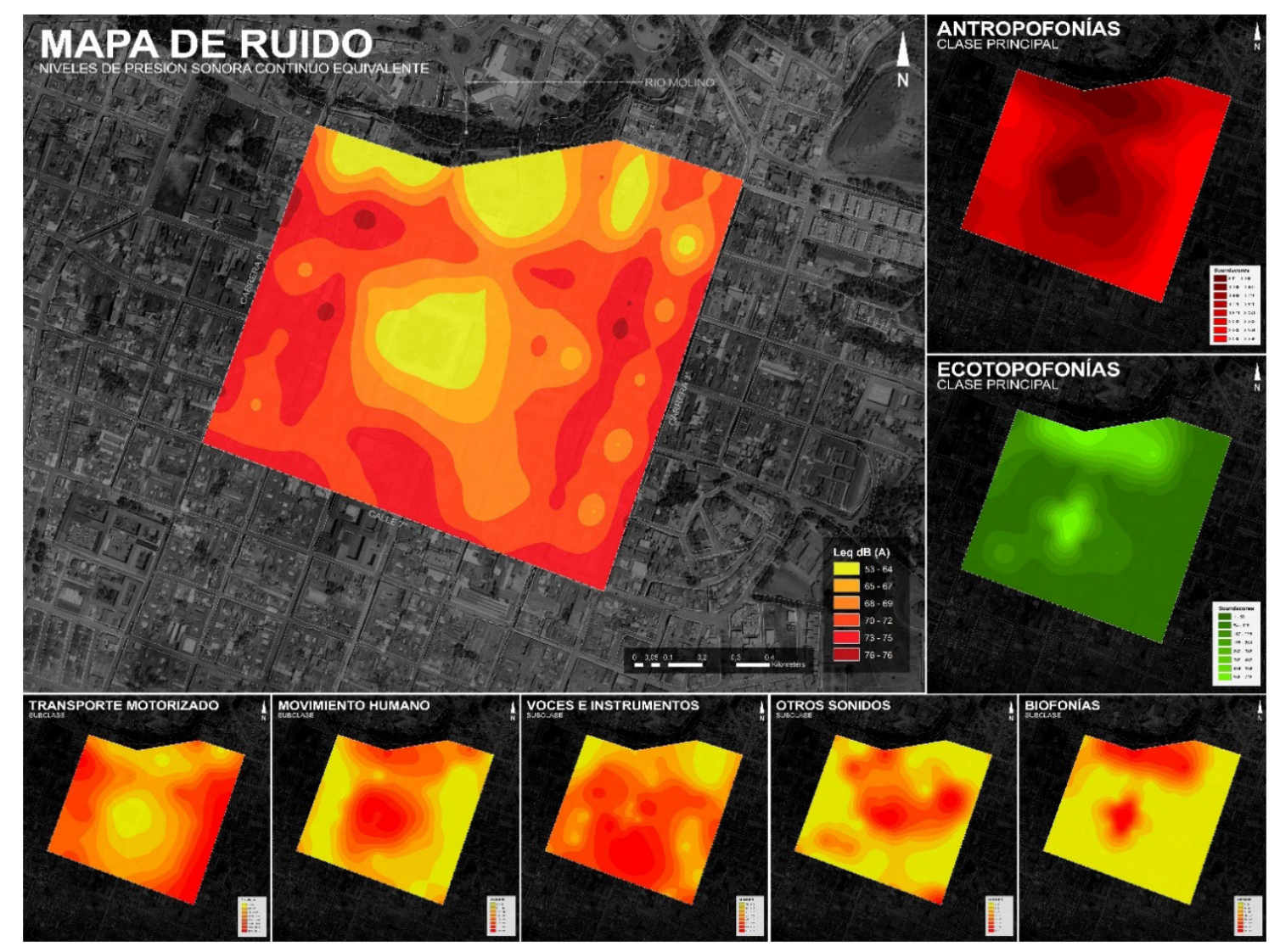

Figura 22: Mapas de las variables integradas del paisaje sonoro urbano. Elaboración propia.

Cabe resaltar que las cartografías sonoras en donde se integran factores físicos y perceptuales del sonido son un método eficaz para visualizar el comportamiento del sonido de la ciudad, la composición general del paisaje sonoro y los indicadores acústicos del sonido en un área urbana determinada.

\section{Información del paisaje sonoro urbano}

La información del paisaje sonoro fue recolectada dentro del perímetro de la U.G.U. de Santo Domingo en el sector histórico de la ciudad de Popayán (Colombia) con el fin de generar cartografías sonoras de carácter integral para su estudio. De esta forma se identificaron los elementos que componen el paisaje sonoro urbano en función de las fuentes sonoras y se relacionó con los parámetros acústicos del sonido.

Al evaluar el paisaje sonoro urbano mediante los mapas en cuestión, se revelaron patrones de comportamiento altamente dinámicos tanto a nivel espacial como temporal. Apoyado en lo propuesto por Liu et al. (2013) se indicó que el comportamiento cambiante del paisaje sonoro en la ciudad se da principalmente por la actividad humana, además la evidencia señala que la actividad del ecosistema natural no deja de ser un factor que tiene influencia en la experiencia sonora de la ciudad.

Los hallazgos de este estudio sugieren que la variabilidad espaciotemporal del paisaje sonoro está 
relacionada con la calidad del entorno acústico urbano en donde no solo hay afectación por niveles que sobrepasan los estándares internacionales de emisión de ruido, sino que también la diversidad del paisaje sonoro y sus patrones de comportamiento convergen hacia el bienestar social de la población.

Son justamente las zonas con altos niveles de contaminación acústica aquellas en las cuales se percibieron paisajes sonoros dominados por el sonido del transporte motorizado, confirmando que el entorno acústico urbano sufre afectación por esta clase de sonido. Según Raimbault y Dubois (2005) el tráfico es uno de los factores que contribuye masivamente a las bajas condiciones sonoras del medio ambiente urbano, razón por la cual surge lo que se conoce como molestia por ruido, no es ruido, son paisajes sonoros dominados por el sonido del motor, como se probó en este estudio.

No obstante las dinámicas cambiantes del paisaje sonoro urbano revelaron algunos escenarios de valor positivo para la población. En ese sentido los planteamientos de Schafer (1993) sobre la búsqueda de experiencias placenteras para el oyente, se reflejaron en los mapas de ecotopofonía como resultado de los sonidos producidos por la naturaleza y que tuvieron mayor incidencia en horas de la mañana. Por tanto la experiencia sonora positiva en la ciudad depende de la dominancia de sonidos generados por la naturaleza, los cuales son típicamente preferidos sobre los sonidos del tráfico o de las maquinas (Nilsson y Berglund, 2005).

Aunque la disminución del nivel de contaminación acústica ha sido el eje principal en la gestión del ruido urbano, no es una acción que necesariamente se relacione con la mejora de la calidad del paisaje sonoro. Es de tener en cuenta que sonidos como voces de personas, risas o pasos ocupando el espacio público de la ciudad, puedan ser sonidos que aporten positivamente al entorno acústico urbano independientemente del nivel de presión sonora. Lo que si es cierto es que definitivamente sonido del motor debe ser removido del paisaje sonoro, si lo que se quiere es velar por el bienestar de la ciudad.

El alcance de este trabajo se delimito en un área urbana con altos índices de diversidad en el uso del suelo, partiendo de la idea de que los paisajes sonoros se componen principalmente de los sonidos producidos por las actividades del entorno urbano, se recomienda entonces que a futuro se amplié la escala espaciotemporal más allá del alcance propuesto en este estudio y de estudios similares como los de Hong y Jeon (2017).

\section{La utilidad del paisaje sonoro urbano}

Una preocupación más centrada en el objeto que en el ser humano ha sido una de las más grandes debilidades de los tomadores de decisiones en la gestión del ruido urbano. En consecuencia se ha descuidado por completo la importancia de la calidad del ambiente, espacio en el cual la introducción de valores positivos para la mejora de las experiencias sonoras de la ciudad puede suscitarse (Raimbault y Dubois. 2005).

Esta investigación apoya la idea del paisaje sonoro como instrumento de planificación urbana, facilitando la prestación de servicios ecosistémicos importantes en la mejora de la calidad de vida de la población. 
Yang y Kang (2005) aseguran que los beneficios del paisaje sonoro dominado por sonidos naturales están directamente asociados a la salud ambiental y por ende con el bienestar social. Por tanto, las entidades locales deben planear estrategias para frenar la degradación del entorno acústico urbano y preservar áreas de interés ambiental que incluyan aspectos de ecología acústica urbana como mecanismo para la creación de ambientes más sanos y habitables para la ciudadanía (Liu et al., 2013).

Se asumió que los entornos urbanos afectan la percepción del paisaje sonoro en varios niveles, por un lado las características del lugar determinan la clase de sonido que se genera y por otro lado la configuración urbana afecta la propagación del sonido creando distintos patrones de comportamiento. En ese sentido, el contexto urbano adyacente es tenido en cuenta como el enlace que combina tanto la información del paisaje sonoro como las características físicas y sociales del lugar. De esta forma, el potencial el paisaje sonoro urbano para implementar prácticas de diseño urbano es de utilidad en el desarrollo sostenible del territorio.

\section{Conclusiones}

Los valores físicos y perceptuales del paisaje sonoro de una pieza urbana fueron registrados y comparados mediante recolección de datos in situ. Principalmente se pensó en examinar la influencia de la variabilidad espaciotemporal del paisaje sonoro en la calidad del entorno urbano, incluyendo la relación de contexto urbano adyacente con todos los aspectos que integran el paisaje sonoro de la ciudad.

Las relaciones de sonoridad entre las clases y subclases del paisaje sonoro son un componente que puede desempeñar un papel fundamental en el ejercicio de la planificación urbana sostenible, ya a que los tomadores de decisiones pueden aunar esfuerzos en el mejoramiento de la calidad de los entornos acústicos de la ciudad. Así, la inclusión de áreas urbanas que propicien sonidos deseados por los habitantes de la ciudad es una directriz que seguir si se quiere contribuir al bienestar social de la población.

De los principales aportes que la dimensión sonora propone al urbanismo es justamente el uso del paisaje sonoro como instrumento de planificación urbana direccionado al planteamiento de modelos de ocupación territorial en donde prevalezcan entornos acústicos de valor positivo para la ciudadanía. Priorizar las áreas urbanas como parques y corredores verdes en los procesos de ciudad sería entonces el camino por seguir. La figura 23 ejemplifica la idea de un sistema de urbano y la consecuente distribución del paisaje sonoro en función de ocupación del suelo urbano. 

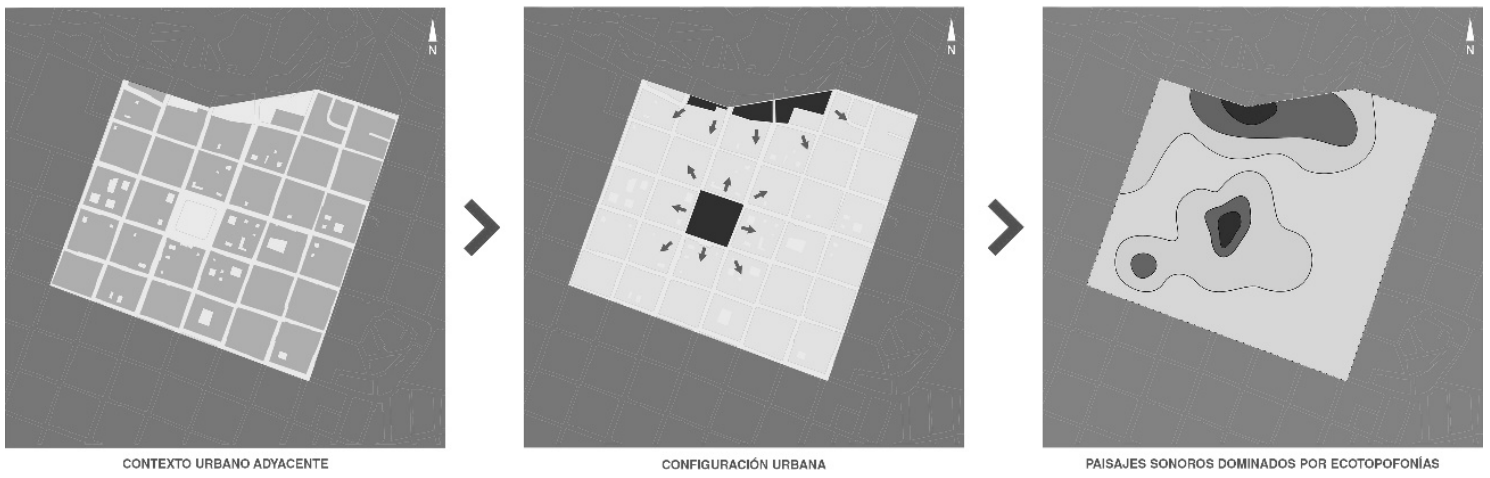

Figura 23: Esquema de un modelo de ocupación territorial acorde al paisaje sonoro. Elaboración propia.

Es importante entonces que las entidades locales tomen cartas en el asunto y se formulen políticas públicas encaminadas a dar soluciones eficaces en función a todos los aspectos que integran el paisaje sonoro urbano, especialmente durante los procesos de ordenamiento territorial. Las ciudades necesitaran siempre del dialogo constante entre sus habitantes y el universo sonoro resultante, por tanto el entorno acústico excesivamente silencioso no puede seguir siendo indicativo de un paisaje sonoro de alta calidad.

Finalmente es de anotar que las intervenciones a los entornos acústicos definitivamente deben ir ligadas a la mejora de la calidad de vida de la población, más aún cuando la dimensión sonora es entendida como parte del sistema territorial en el que se encuentran los habitantes de la ciudad a diario. Es fundamental entonces que desde acciones urbanísticas se promueva la mejora de la impresión global del paisaje sonoro urbano, teniendo en cuenta siempre unos valores de reciprocidad y un sentido ético para todos los actores del territorio.

\section{Agradecimientos}

Este material se desarrolló en el marco del proyecto de Investigación y Desarrollo de la Planificación Urbana Sostenible en el Cauca - Estudio de Caso Popayán, financiado por el Fondo de Ciencia, Tecnología e Innovación del Gobierno de Colombia y ejecutado por la Institución Universitaria Colegio Mayor del Cauca.

El Centro De Estudios Urbanos de la institución respaldo con mucha convicción el trabajo, por lo cual agradecemos a su fundador, el PhD Francisco Zúñiga, quien con su experiencia docente ayudo considerablemente en la investigación, y al director del proyecto, el Arq. German Chamorro, por su desinteresado apoyo hacia todas las cuestiones que implico llevar a cabo este estudio.

\section{Referencias}

- $\quad$ Agnew, J. (2011) Space and place. Handbook of Geographical Knowledge, Londres, 316-331.

- Bahal, S. y Tamer-Bayazit, N. (2017). Soundscape research on the Gezi Park-Tunel Square route. Applied Acoustics, 116, 260-270. doi: 10.1016/j.apacoust.2016.10.002 
- Brown, A. L., Kang, J. y Gjestland, T. (2011). Towards standardization in soundscape preference assessment. Applied Acoustics, 72(6), 387-392. doi: 10.1016/j.apacoust.2011.01.001

- $\quad$ Bruce, N. S. y Davies, W. J. (2014). The effects of expectation on the perception of soundscapes. Applied Acoustics, 85, 1-11. doi: 10.1016/j.apacoust.2014.03.016

- $\quad$ Can, A., Leclercqa, L. y Lelongb, J. (2008). Dynamic estimation of urban traffic noise: Influence of traffic and noise source representations. Applied Acoustics, 69(10), 858-867. doi: 10.1016/j.apacoust.2007.05.014

- $\quad$ Carles, J. L., Barrio, I. L. y de Lucio, J. V. (1999). Sound influence on landscape values. Landscape and Urban Planning, 43(4), 191-200. doi: 10.1016/S0169-2046(98)00112-1

- $\quad$ Cerwén, G. (2016). Urban soundscapes: A quasi-experiment in landscape architecture. Landscape Research, 41(5), 481-494. doi: 10.1080/01426397.2015.1117062

- Davies, W. J., Adams, M. D., Bruce, N. S., Cain, R., Carlyle, A., Cusack, P., et al. (2013). Perception of soundscapes: An interdisciplinary approach. Applied Acoustics, 74(2), 224-231. doi: 10.1016/j. apacoust.2012.05.010

- Geraghty, D. y O'Mahony, M. (2016). Investigating the temporal variability of noise in an urban environment. International Journal of Sustainable Built Environment, 5(1), 34-45. doi: 10.1016/j. ijsbe.2016.01.002

- Hao, Y. y Kang, J. (2014). Influence of mesoscale urban morphology on the spatial noise attenuation of flyover aircrafts. Applied Acoustics, 84, 73-82. doi: 10.1016/j.apacoust.2013.12.001

- Harman, B. I., Koseoglu, H. y Yigit, C. O. (2016). Performance evaluation of IDW, Kriging and multiquadric interpolation methods in producing noise mapping: A case study at the city of Isparta, Turkey. Applied Acoustics, 112, 147-157. doi: 10.1016/j.apacoust.2016.05.024

- $\quad$ Hong, J. Y. y Jeon, J. Y. (2017). Exploring spatial relationships among soundscape variables in urban areas: A spatial statistical modelling approach. Landscape and Urban Planning, 157, 352-364. doi: 10.1016/j. landurbplan.2016.08.006

- Jennings, P. y Cain, R. (2013). A framework for improving urban soundscapes. Applied Acoustics, 74(2), 293-299. doi: 10.1016/j.apacoust.2011.12.003

- Jeon, J. Y. y Hong, J. Y. (2015). Classification of urban park soundscapes through perceptions of the acoustical environments. Landscape and Urban Planning, 141, 100-111. doi: 10.1016/j.landurbplan.2015.05.005

- Joo, W., Gage, S. H., \& Kasten, E. P. (2011). Analysis and interpretation of variability in soundscapes along an urban-rural gradient. Landscape and Urban Planning, 103(3), 259-276.

- $\quad$ Kang, J. Aletta, F., Gjestland, T. T., Brown, L. A., Botteldooren, D., Schulte-Fortkamp, B., et al. (2016). Ten questions on the soundscapes of the built environment. Building and Environment, 108, 284-294. doi: 10.1016/j.buildenv.2016.08.011

Leus, M. y Herssens, J. (2015). The soundscapes of Antwerp: a study on the acoustic genius loci. 
Energy Procedia, 78, 25-30. doi: 10.1016/j.egypro.2015.11.109

- Liu, F. y Kang, J. (2016). A grounded theory approach to the subjective understanding of urban soundscape in Sheffield. Cities, 50, 28-39. doi: 10.1016/j.cities.2015.08.002

- $\quad$ Liu, J., Kang, J., Luo, T., Behm, H. y Coppack, T. (2013). Spatiotemporal variability of soundscapes in a multiple functional urban area. Landscape and Urban Planning, 115, 1-9. doi: 10.1016/j.landurbplan.2013.03.008 - Ministerio de Cultura de Colombia. (2010). Resolución 2432. Por la cual se aprueba el Plan Parcial de Manejo y Protección del Sector Antiguo de Popayán. Diario Oficial, 47684, 104.

- Mohammadi, G. (2009). An investigation of community response to urban traffic noise. Global Perspective for Competitive Enterprise, Economy and Ecology, 673-680.

- Nilsson, M. E. y Berglund, B. (2005). Assessment of outdoor soundscapes in quiet areas. The Journal of the Acoustical Society of America, 117(4), 2592-2592. doi: 10.1121/1.4777308

- $\quad$ Organización Mundial De La Salud. (1999). Guías para el ruido. Switzerland, Geneva; OMS.

- $\quad$ Pijanowski, B. C., Farina, A., Gage, S. H., Dumyahn, S. L. y Krause, B. L. (2011). What is soundscape ecology? An introduction and overview of an emerging new science. Landscape Ecology, 26(9), 1213-1232. doi: 10.1007/s10980-011-9600-8

- Raimbault, M. y Dubois, D. (2005). Urban soundscapes: Experiences and knowledge. Cities, 22(5), 339-350. doi:10.1016/j.cities.2005.05.003

- Rehan, R. M. (2016). The phonic identity of the city urban soundscape for sustainable spaces. HBRC Journal, 12(3), 337-349. https://doi.org/10.1016/j.hbrcj.2014.12.005

- $\quad$ Schaeffer, P., Mâche, F. B., Philippot, M., Bayle, F., Ferrari, L., Malec, I., et al. (1967). La musique concrète. Presses universitaires de France.

- Schafer, R. M. (1993). The soundscape: Our sonic environment and the tuning of the world. Simon and Schuster.

- Schiewe, J. y Weninger, B. (2013). Visual encoding of acoustic parameters-framework and application to noise mapping. The Cartographic Journal, 50(4), 332-344. doi: 10.1179/1743277412Y.0000000026

- $\quad$ Simmel, G. (1903). The metropolis and mental life.

- $\quad$ Stanners, D. y Bourdeau, P. (1995). Europes environment; the Drobris assessment. Copenhagen: European Environment Agency.

- $\quad$ Stoter, J., Kluijver H. y Kurakula, V. (2008). 3D noise mapping in urban areas. International Journal of Geographical Information Science, 22(8), 907-924. doi: 10.1080/13658810701739039

- Truax, B. (2006). La composición de paisajes sonoros como música global. Recuperado de: https:// goo.gl/VzGf56

- Tsai, K. T., Lin, M. D., y Chen, Y. H. (2009). Noise mapping in urban environments: A Taiwan study. 
Applied Acoustics, 70(7), 964-972. doi: 10.1016/j.apacoust.2008.11.001

- Watts, G. R. y Pheasant, R. J. (2015). Tranquillity in the Scottish Highlands and Dartmoor National Park-The importance of soundscapes and emotional factors. Applied Acoustics, 89, 297-305. doi: 10.1016/j. apacoust.2014.10.006

- Yang, W., \& Kang, J. (2005). Soundscape and sound preferences in urban squares: a case study in Sheffield. Journal of Urban Design, 10(1), 61-80.

- Zannin, P. H. T., Engel, M. S., Fiedler, P. E. K. y Bunn, F. (2013). Characterization of environmental noise based on noise measurements, noise mapping and interviews: A case study at a university campus in Brazil. Cities, 31, 317-327. doi: 10.1016/j.cities.2012.09.008 